Florida International University FIU Digital Commons

8-10-2016

\title{
Synthetic Studies Toward a Novel Hydroxylamine of Potential Utility in the Preparation of Mitochondriotropic Nitrones
}

Gloria Patricia Perez

gpere159@fiu.edu

DOI: $10.25148 /$ etd.FIDC001182

Follow this and additional works at: https://digitalcommons.fiu.edu/etd

Part of the Organic Chemistry Commons

\section{Recommended Citation}

Perez, Gloria Patricia, "Synthetic Studies Toward a Novel Hydroxylamine of Potential Utility in the Preparation of Mitochondriotropic Nitrones" (2016). FIU Electronic Theses and Dissertations. 3052.

https://digitalcommons.fiu.edu/etd/3052 


\section{FLORIDA INTERNATIONAL UNIVERSITY}

Miami, Florida

SYNTHETIC STUDIES TOWARD A NOVEL HYDROXYLAMINE OF POTENTIAL UTILITY IN THE PREPARATION OF MITOCHONDRIOTROPIC NITRONES

A thesis submitted in partial fulfillment of

the requirements for the degree of

MASTER OF SCIENCE

in

CHEMISTRY

by

Gloria P. Perez 
To: Dean Michael R. Heithaus

College of Arts, Sciences and Education

This thesis, written by Gloria P Perez, and entitled Synthetic Studies Toward a Novel Hydroxylamine of Potential Utility in the Preparation of Mitochondriotropic Nitrones, having been approved in respect to style and intellectual content, is referred to you for judgment.

We have read this thesis and recommend that it be approved.

Watson Lees

Stainslaw Wnuk

David Becker, Major Professor

Date of Defense: August 10, 2016

The thesis of Gloria P Perez is approved.

Dean Michael R. Heithaus College of Arts, Sciences and Education

Andrés G. Gil Vice President for Research and Economic Development and Dean of the University Graduate School

Florida International University, 2016 
(C) Copyright 2016 by Gloria P Perez

All rights reserved. 


\section{DEDICATION}

I dedicate this work to the man that instilled me love for science, my mentor, my friend, my inspiration, my hero and one of the most brilliant minds I ever known: my father Jorge Perez. Daddy without you, this work would not have been possible. 


\section{ACKNOWLEDGMENTS}

I would like to thank Professor David Becker for giving me the honor to be part of his lab. My experience working in his lab, not only contributed to my professional development as a chemist but also to my personal growth. Without his patience, understanding and valuable mentorship this work would not have been completed.

I would also like to take this opportunity to thank my committee members: Dr. Lees

and Dr. Wnuk for their service and support throughout my studies at this institution. Equally important, I would like to extend my gratitude to Lilia San Miguel and her collaborators, members of Dr. Wnuk's lab, and my lab mate Nagaraju for their unconditional support.

Last but not least, I would like to thank my parents and my husband. The work accomplished in this research is the sole reflection of your support, love, patience and encouragement. Thank you all for believing in me and for reminding me that anything you set your mind to do you can accomplish. 


\begin{abstract}
OF THE THESIS
SYNTHETIC STUDIES TOWARD A NOVEL HYDROXYLAMINE OF POTENTIAL UTILITY IN THE PREPARATION OF MITOCHONDRIOTROPIC NITRONES
\end{abstract}

by

Gloria P. Perez

Florida International University, 2016

Miami, Florida

\title{
Professor David Becker, Major Professor
}

Mitochondrial dysfunction and oxidative stress have been linked with many pathological conditions. Studies suggest that antioxidants able to accumulate in the inner regions of the mitochondria as well as possessing the chemical entities to cross membranes such as the blood brain barrier, could potentially overcome many of the limitations found with current antioxidants.

Nitrones have demonstrated great antioxidant potential due to their free radical scavenging properties, cardio and neuro-protective activities. In this research, several synthetic studies were performed with the aim of synthesizing a novel hydroxylamine of potential utility for the preparation of mitochondria targeted nitrones. These studies resulted in the generation of a synthetic intermediate, $N$-(3-(1H-imidazol-1-yl)propyl)2,2,3-trimethyl-3-nitrobutaminde, that although lacking the desired hydroxyl- amino functionality, this intermediate is, in theory, an immediate precursor to a molecule that is endowed with the structural characteristics to deliver tethered nitrones to the inner regions of the mitochondria. 


\section{TABLE OF CONTENTS}

CHAPTER

PAGE

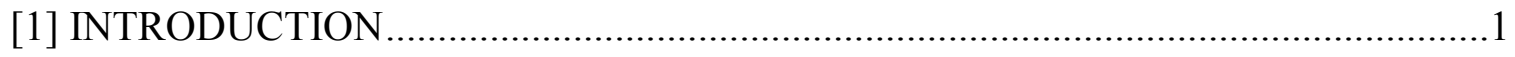

[1.1] Mitochondrial Oxidative Damage and Free Radical Generation ........................2

[1.2] Free Radical Involvement in Human Pathologies ............................................

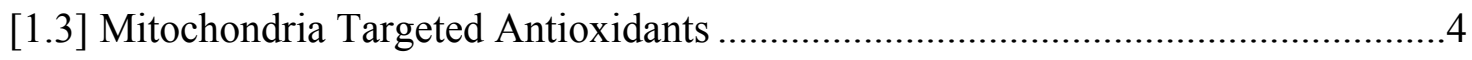

[1.3.1] TPP+ Conjugated Vitamin E and CoQ antioxidants .................................5

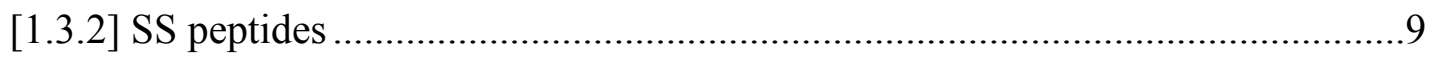

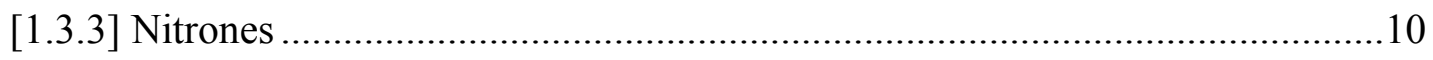

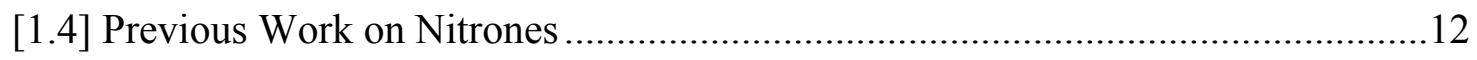

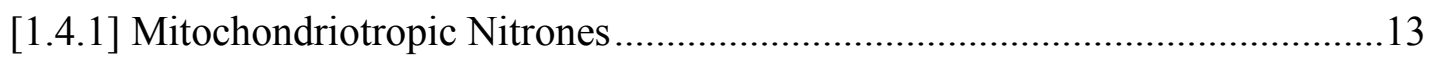

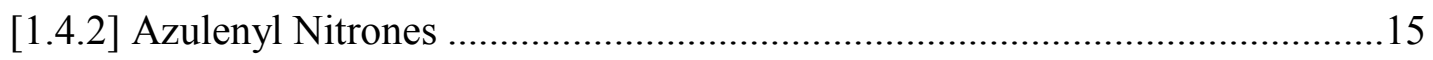

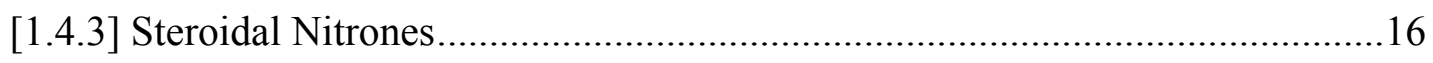

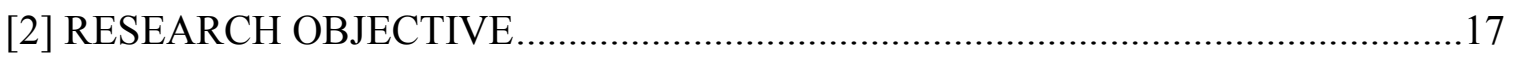

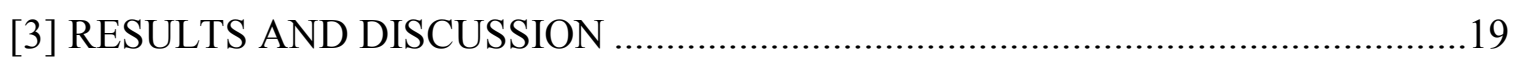

[3.1] Direct amidation of compound (3) with hexylamine .......................................19

[3.2] Catalyzed amidation of compound (3) with imidazole-amine (6) ......................21

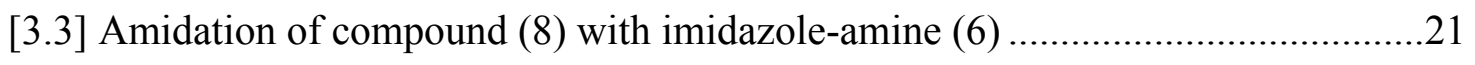

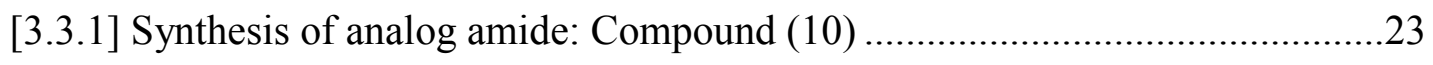

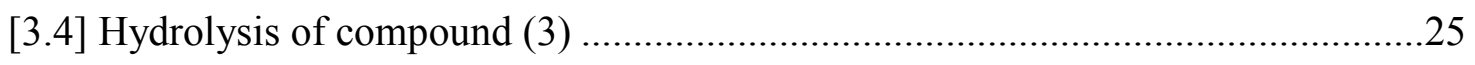

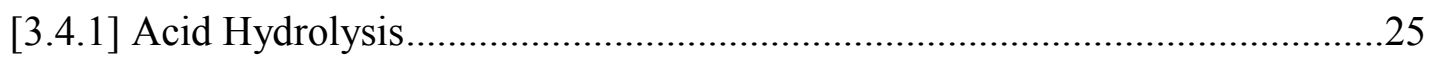

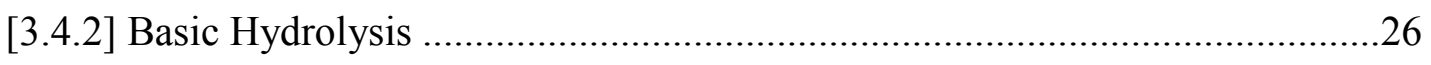

[3.5] DCC coupling of compound (11) with imidazolamine (6) ...............................28

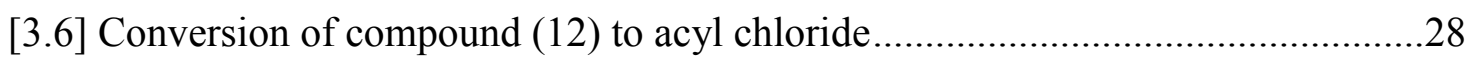

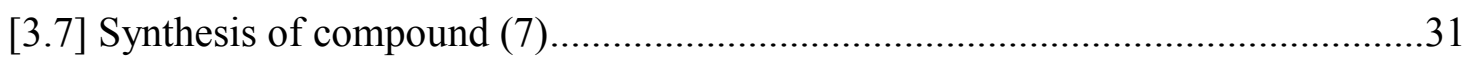

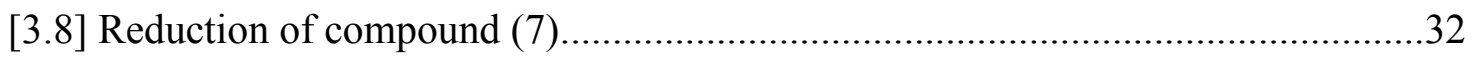

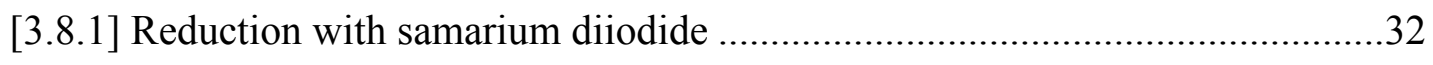

[3.8.2] Reduction with ammonium chloride and zinc powder................................33

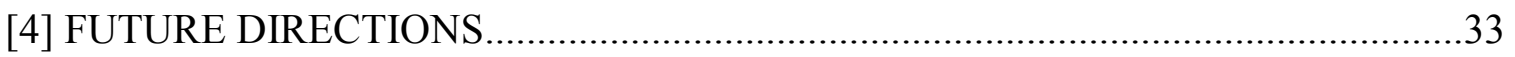

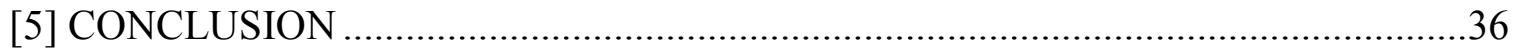




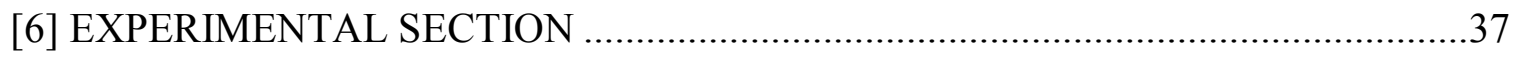

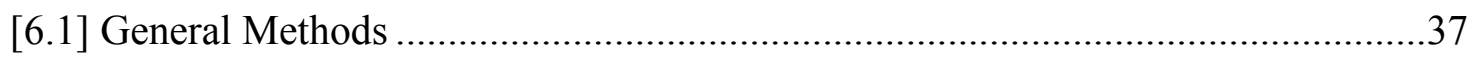

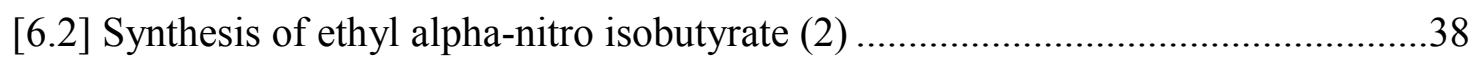

[6.3] Synthesis of ethyl 2,2,3-trimethyl-3-nitrobutanoate (3).......................................39

[6.4] Acid hydrolysis ethyl 2,2,3-trimethyl-3-nitrobutanoate (3) ................................40

[6.5] Basic hydrolysis ethyl 2,2,3-trimethyl-3-nitrobutanoate (3) ...............................40

[6.6] Synthesis of $N$-(3-1H-imidazol-1-yl)-2-bromo-2-methylpropanamide (9) ...........41

[6.7] Synthesis of $N$-(3-(1H-imidazol-1-yl)propyl)-2,2,3-trimethyl-3-

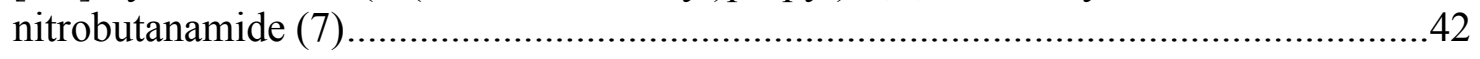

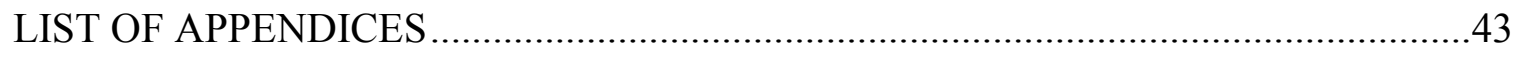

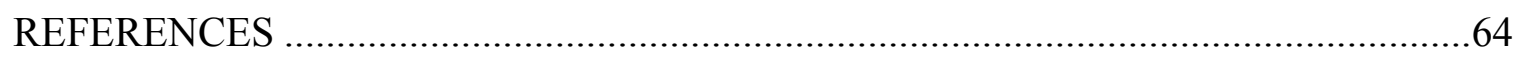




\section{LIST OF FIGURES}

\section{FIGURE}

PAGE

Figure 1. Formation and dismutation of reactive oxygen species. ….............................. 3

Figure 2. Mitochondria targeted antioxidants: mitovitE and mitoQ ............................ 6

Figure 3. Uptake of TPP cations through the phospholipid membrane.......................... 7

Figure 4. Electropositive carbons present on carbonyl compounds and nitrones............ 11

Figure 5. Generation of aminoxyl spin adduct......................................................... 11

Figure 6. Spin adduct formation from PBN nitrone .................................................. 12

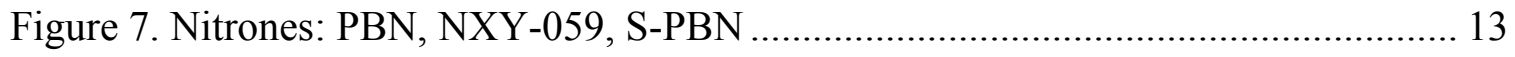

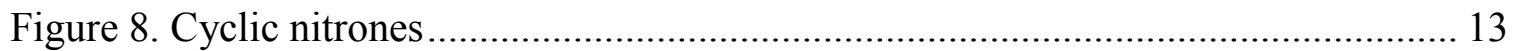

Figure 9. Structures of MitoPBN, MitoBMPO, MitoDEPMPO .................................... 14

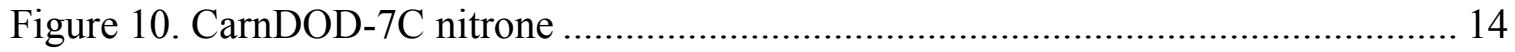

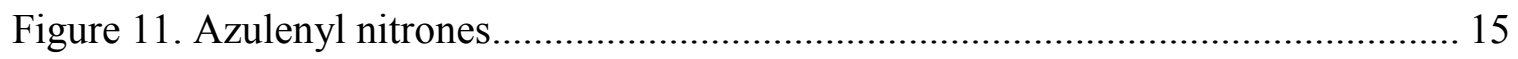

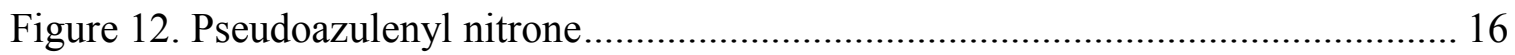

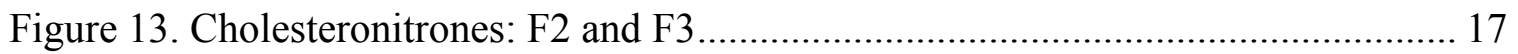

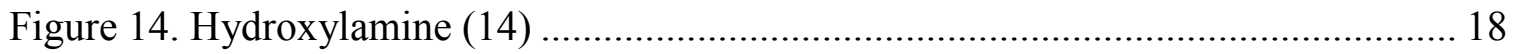

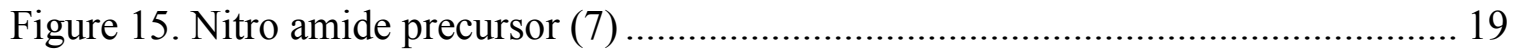




\section{LIST OF ABBREVIATIONS}

$\mathrm{AD}$

Alzheimer's disease

ALS

Amyotrophic lateral sclerosis

ATP adenosine triphosphate

AZN azulenyl nitrone

$\mathrm{CH} 3 \mathrm{CN}$ acetonitrile

DCC N,N'-Dicyclohexylcarbodiimide

DEPMPO 5-diethyoxyphosphoryl-5-methyl-1-pyroline N-oxide

DMF N,N-dimethylformamide

DMPO 5,5-dimethyl-3,4-dihydro-2 H-pyrrole N-oxide

DMSO dimethyl sulfoxide

DNA. deoxyribonucleic acid

EMPO 5-ethoxycarbonyl-5-methyl-1-pyroline $\mathrm{N}$-oxide

EPR electron paramagnetic resonance

ESR electron spin resonance

ETC electron transport chain

$\mathrm{EtOH}$ ethanol GPx glutathione peroxidase 
$\mathrm{HCl}$ Hydrochloric acid

$\mathrm{HO}^{*}$ hydroxyl radical $\mathrm{HOO}^{*}$ hydroperoxyl radical HPLC high performance liquid chromatography

HRMS high resolution mass spectrometry

$\mathrm{MeOH}$ methanol

NADH nicotinamide adenine dinucleotide hydride

NADHP nicotinamide adenine dinucleotide phosphate

NMR nuclear magnetic resonance

NOS nitric oxide synthase

${ }^{1} \mathrm{O}_{2}$ singlet oxygen

$\mathrm{O}_{2}{ }^{--}$ superoxide radical anion

$\mathrm{ONOO}^{-}$ peroxynitrite anion PBN $\alpha-N$-phenyl- $N$-tert-butylnitrone

PD Parkinson's disease ROS reactive oxygen species 
RT room temperature

SOD superoxide dismutase

STAZN. stilbazulenyl nitrone

THF tetrahydrofuran

TLC thin layer chromatography

UV-vis ultraviolet visible 


\section{LIST OF SCHEMES}

SCHEME

PAGE

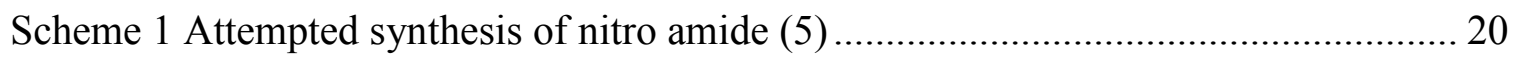

Scheme 2. Attempted synthesis of nitro amide (7) precursor using $\mathrm{MgCl}_{2} \ldots \ldots \ldots \ldots \ldots \ldots . . . . . .21$

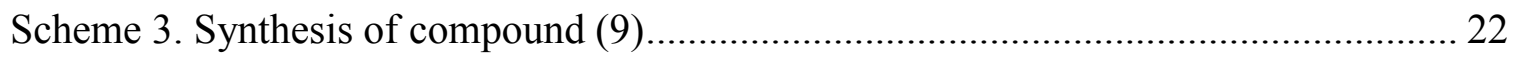

Scheme 4. Attempted synthesis of nitro amide precursor through compound (9).......... 23

Scheme 5. Proposed synthetic approach for nitro amide precursor using

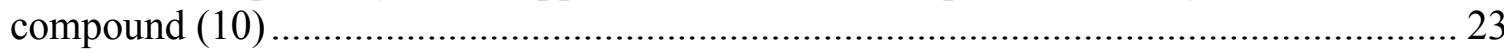

Scheme 6 . Acid hydrolysis of compound (3) .................................................... 25

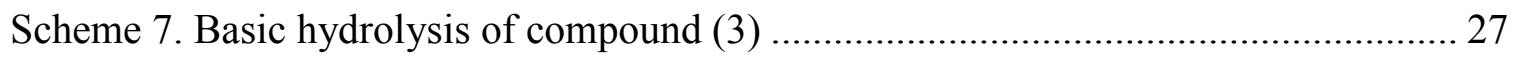

Scheme 8. Synthetic scheme of DCC catalyzed amidation of compound (11) ............... 28

Scheme 9. General approach for acyl chloride formation using carboxylate (12) .......... 29

Scheme 10. Attempted synthesis of nitro amide (7) using sulfuryl chloride ................... 30

Scheme 11. Attempted synthesis of nitro amide (7) using oxalyl chloride .................... 30

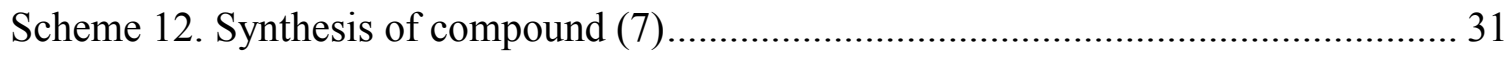

Scheme 13. Proposed synthetic strategy for compound (17) ..................................... 35

Scheme 14. Proposed synthetic strategy through direct coupling of compound (7) ........ 36 


\section{[1] INTRODUCTION}

Many studies have suggested that mitochondrial dysfunction is associated with several pathological conditions such as Alzheimer's disease (AD), ischemic stroke, Parkinson's disease (PD), ischemia reperfusion, and amyotrophic lateral sclerosis (ALS) among other neurodegenerative diseases ${ }^{1}$. The mitochondrion is the site of energy production inside the cell and is constantly exposed to oxidative damage ${ }^{1,2}$. As the power source of the cell, the mitochondrion is a major source of reactive oxygen species (ROS) such as superoxide anion radical, $\mathrm{O}_{2}{ }^{\bullet-}$, and hydrogen peroxide, $\mathrm{H}_{2} \mathrm{O}_{2}$. These oxygen species can lead to the formation of even more deleterious species like hydroxyl radicals, $\cdot \mathrm{OH}^{1,3}$. Studies have revealed that these ROS can cause damage to mitochondrial components as well as the triggering of harmful processes inside the cell ${ }^{3,4}$.

Previous studies on animal models have shown that mitochondrial protection against oxidative stress is crucial in attenuating or delaying the progress of several neuronal pathologies ${ }^{5}$. Because there is great evidence that mitochondrial dysfunction is intimately associated with a myriad of cellular processes that lead to cell death and necrosis, mitochondria targeted antioxidants can serve as promising therapeutic agents ${ }^{5,6}$.

The work herein describes the strategies used to design mitochondria targeted nitrones as well as the role of nitrone chemistry in biological systems. In the present research, several studies were performed in order to synthesize $\mathrm{N}$-(3-1H-imidazol-1yl)propyl)-3-(hydroxyamino)-2,2,3-trimethylbutamide (14), a novel hydroxylamine of potential utility for the preparation of mitochondria targeted nitrones. Through this scientific endeavor, a nitro amide precursor, $N$-(3-(1H-imidazol-1-yl)propyl)-2,2,3- 
trimethyl-3-nitrobutaminde (7) was successfully synthesized. Although lacking the desired hydroxyl- amino functionality, this intermediate appears to be a viable precursor to a molecule that is endowed with the structural characteristics to deliver tethered nitrones to the inner regions of the mitochondria. Therefore, the work presented in this research entails the discussion of the therapeutic applications of nitrones and future synthetic methodologies that can be utilized to create a new class of pseudoazulenyl mitochondriotropic nitrones.

[1.1] Mitochondrial Oxidative Damage and Free Radical Generation

One of the most important functions of the mitochondrion is the production of ATP, which provides the energy for nearly all biological processes inside the cell. However, this energy generation could trigger unwanted events such as the production of ROS. Under normal aerobic conditions the mitochondria produces $90 \%$ of the cellular energy through the electron transport chain $(\mathrm{ETC})^{7,8,9}$. During this process about $2 \%$ of electrons leak out, causing a downstream of free radical reactions that, if unregulated, can interfere with mitochondrial function ${ }^{7,10}$.

The antioxidant defense system of the mitochondria contains several enzymes that help maintain the cellular homeostasis. For instance, superoxide dismutase (SOD) detoxifies superoxide species produced by electron leakage from the ETC and forms $\mathrm{H}_{2} \mathrm{O}_{2}$. Glutathione peroxidase (GPx) is another antioxidant enzyme that converts $\mathrm{H}_{2} \mathrm{O}_{2}$ to water. Detoxification of $\mathrm{H}_{2} \mathrm{O}_{2}$ prevents the formation of hydroxyl radicals $(\mathrm{OH})$ and hydroxide ions $(\mathrm{OH})$, which can result in irreversible oxidative damage to the cel1 ${ }^{7,10-12}$. The Fenton 
reaction illustrates the production of this deleterious hydroxyl radical by the reaction of unquenched $\mathrm{H}_{2} \mathrm{O}_{2}$ with metal ions such as iron (Figure 1).

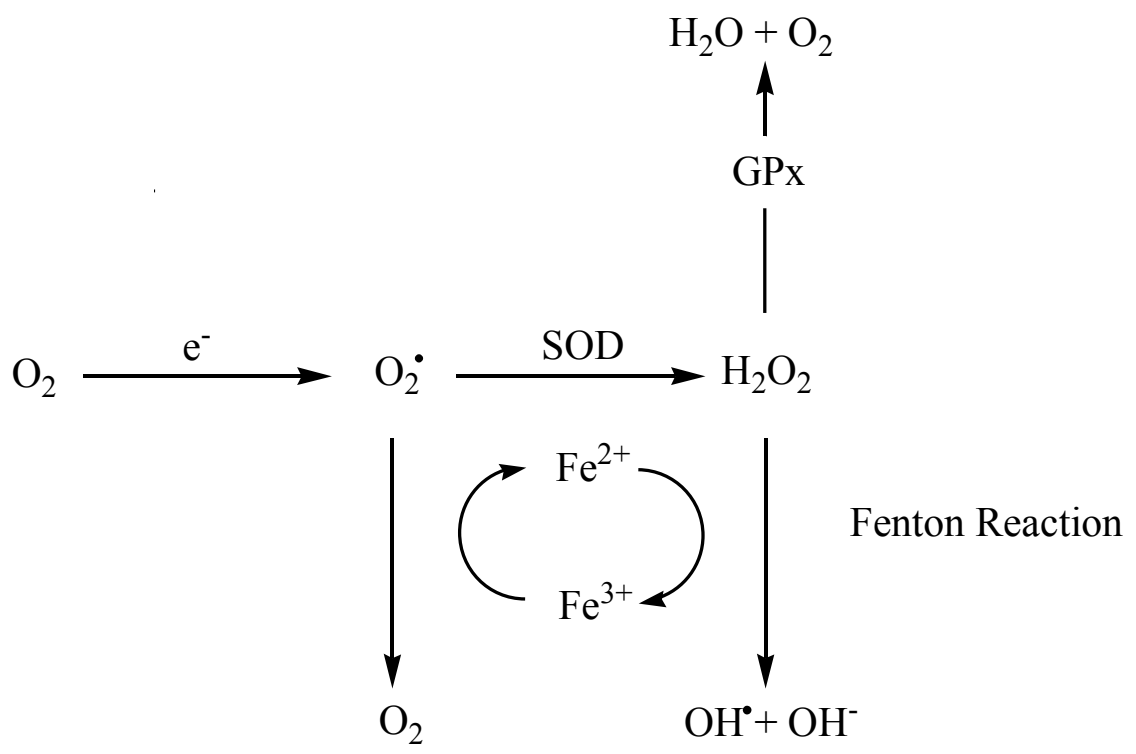

Figure 1. Formation and dismutation of reactive oxygen species. (Adapted from Oyewole et $\mathrm{al}^{7}$ )

Despite the protective action of these endogenous antioxidants, enhanced production of ROS can result in a redox imbalance that leads to oxidative stress and mitochondrial dysfunction. Overproduction of free radicals generates a cascade of calamitous events to the cell such as induced apoptosis, DNA injury, and protein and tissue damage among others ${ }^{3,7,10}$. It is important to note, however, that regulated free radical production has a crucial role in biological processes such as signal transduction, inflammation, gene transcription and more ${ }^{3,7,10}$.

[1.2] Free Radical Involvement in Human Pathologies

A strong body of evidence has shown that oxidative damage is present in a wide range of pathologies ${ }^{13-15}$. Whether oxidative damage is a cause or consequence of a specific 
pathology is unknown ${ }^{7,10}$. Neurodegenerative disorders such as Parkinson's disease (PD), Alzheimer's disease (AD) and Amyotrophic lateral sclerosis (ALS) have displayed increased indices of ROS in postmortem brain tissues ${ }^{10}$. Also, common markers of oxidative damage such as 4-hydroxynonenal (4-HNE), malondialdehyde (MDA), and protein nitration among others have as well been identified. It is hypothesized that oxidative stress is strongly associated with neuronal cell death that takes place in these neurodegenerative conditions ${ }^{3,13,16}$.

Free radicals have also been involved in chemical carcinogenesis, which includes initiation, promotion and progression stages. The activation of many of these steps is postulated to involve free radical intermediates ${ }^{17,18}$. For example, the free radical mediated oxidation of a polycyclic aromatic hydrocarbon to an electrophilic species can generate mutations by binding to DNA. If DNA is damaged, a myriad of events will trigger aberrant processes inside the cell including apoptosis. Uncontrolled program cell death can eliminate healthy cells that could otherwise keep homeostasis in the affected tissues. Another piece of evidence suggesting free radical involvement in cancer is the role of radical scavengers and/or antioxidants in protecting cells from tumor formation. In addition, the pro-inflammatory state associated with tumor development encompasses higher concentrations of endogenous radicals such as superoxide ${ }^{17,19,20}$.

\section{[1.3] Mitochondria Targeted Antioxidants}

Mitochondria carry out imperative metabolic functions that include energy biogenesis, redox signaling, heme biosynthesis, iron-sulfur center assembly, as well as playing important roles in the process of ageing ${ }^{1,6,21}$. If mitochondrial function is impaired 
and ATP production is compromised, necrotic cell death can occur as a result of disruption of cell functions, tissues and organs ${ }^{22}$. Thus, it is reasonable to think that mitochondrial dysfunction resulting from oxidative damage is highly associated with the etiology of countless human pathologies. In fact, there is evidence that imbalanced metabolism and overproduction of ROS can cause oxidative damage to biomolecules (DNA, lipids, proteins) resulting in worsening a range of disorders such as cancer, stroke, chronic inflammation and neurodegenerative diseases $22,17,23$. Therefore, targeting antioxidants to mitochondria is a promising approach to prevent or delay progression of these conditions $^{24,25}$.

Natural antioxidants such as green tea (polyphenols), vitamin E and coenzyme Q (Co Q) have shown protective effects in animal models of PD, AD and ALS but have not shown clinical significant benefits in human trials ${ }^{5,26}$. One possible explanation for these poor outcomes is the lack of antioxidant accumulation inside the mitochondria. For example, Vitamin E and CoQ are highly lipophilic and failed to accumulate inside the mitochondria, which are the sites where most ROS are generated ${ }^{5,6,22}$.

Using the aforementioned rationale, several antioxidants have been engineered to target the mitochondria and these include but are not limited to: triphenylphosphonium $(\mathrm{TPP}+)-$ conjugated vitamin E, CoQ, nitrones and SS peptides. These compounds will be discussed in detail in the following section:

\section{[1.3.1] TPP + Conjugated Vitamin E and CoQ antioxidants}

Inspired by the work of Dr. Skulachev and coworkers who first introduced the use of the triphenylphosphonium (TPP) cation to determine mitochondrial membrane potential, 
the groups of Murphy and Smith have been able to synthesize antioxidants that accumulate inside the mitochondrion using this cationic moiety. The lipophilic antioxidants that were conjugated to the TPP cation were coenzyme Q (MitoQ) and vitamin E (MitovitE) (Figure $2)^{6,22,27}$. The accumulation of these compounds inside the inner regions of the mitochondrion is dependent on the gradient potential across the mitochondrial inner membrane $e^{6,5,22}$.<smiles>COC1=C(OC)C(=O)C(CCCCCCCCCC[P+](c2ccccc2)(c2ccccc2)c2ccccc2)=C(C)C1=O</smiles><smiles>Cc1c(C)c2c(c(C)c1O)CCC(C)(C)O2</smiles>

Figure 2. Mitochondria targeted antioxidants: mitovitE and mitoQ.

Lipophilic cations have the ability to cross the lipid bilayers of the membrane because of their hydrophobic nature and are delivered inside using the negative potential that is generated through the ATP production. The dynamics of this process can be explained by the Born energy equation:

$$
W_{B}=\frac{339 Z^{2}}{r}
$$


The Born energy is represented by WB in $\mathrm{kJ} / \mathrm{mol}$ for a cation with $\mathrm{Z}$ (charge) and $\mathrm{r}$ (radius) of the cation. The equation depicts the activation energy required to move the cation from the aqueous phase to the hydrophobic core of the membrane. The Born energy is inversely proportional to the radius of the cation. Thus, the large radius of the TPP cation results in a small enthalpy required to move the cation into the membrane ${ }^{22,28}$. The increase in hydrophobicity of these positively charged ions facilitates crossing of the phospholipid bilayer relative to other cations ${ }^{22,29,30}$.

In order to penetrate the center of the membrane the cations need to overcome the repulsive electrostatic forces in the external compartment, which is positively charged. The electrostatic repulsion is offset by the attractive hydrophobic forces that increase the entropy favoring the movement across the membrane ${ }^{22,28}$ (Figure 3 ).

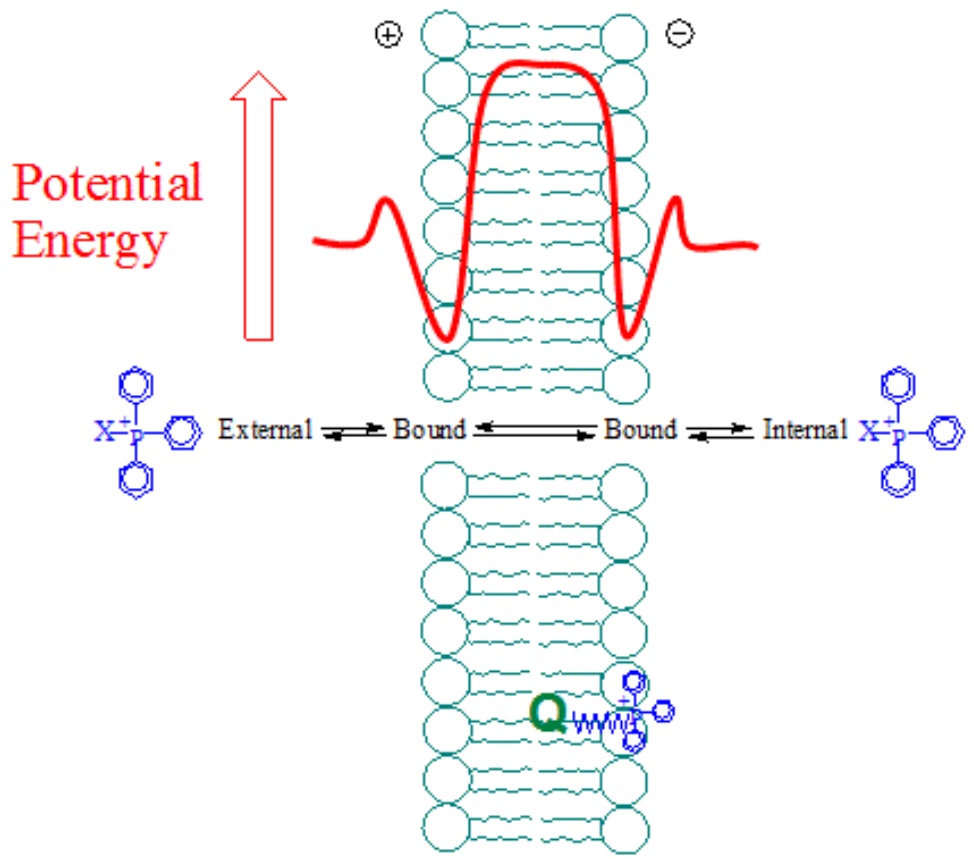

Figure 3. Uptake of TPP cations through the phospholipid membrane. (Adapted from Murphy et al. ${ }^{22}$ ) 


\section{[1.3.1.1] MitoQ}

MitoQ is a derivative of mitochondrial ubiquinone linked to a TPP moiety. Within the mitochondria, MitoQ is accumulated in the inner membrane serving as a 2-electron acceptor from complex 1 or $2^{31,32}$. Subsequently, the quinone is reduced to ubiquinol, which transfers the electrons to complex 3. Both forms (ubiquinone/ubiquinol) posses antioxidant properties. Ubiquinol in particular is able to donate a hydrogen atom from one of its hydroxyl groups to a lipid peroxyl radical thus preventing lipid peroxidation inside the mitochondrial membrane $e^{31,33,34,35}$. MitoQ was found to accumulate in the mitochondria and most importantly prevented further radical toxicity from peroxide by converting $\mathrm{H}_{2} \mathrm{O}_{2}$ to $\mathrm{H}_{2} \mathrm{O}$ and $\mathrm{O}_{2}{ }^{31}$.

The work of Murphy and Smith have also revealed important information about the structural characteristics of an effective mitochondriotropic antioxidant. They analyzed several derivatives of MitoQ varying the number of carbons in their linker chains ${ }^{36}$. It was found that the carbon chain length affected the efficacy of the MitoQ antioxidants. The MitoQ compounds having short carbon chains were less effective antioxidants ${ }^{22,21,36}$. This carbon chain limitation may be explained by the restricted access of the short chain compounds in reaching the hydrophobic core of the membrane ${ }^{22}$.

The modified mitochondrial ubiquinone, MitoQ, was studied in cultured fibroblasts from Friedreich Ataxia (FRDA) patients. The pathology of FRDA is characterized by an autosomal disease described by increased ROS and a defective mitochondrial protein, Frataxin ${ }^{10,37,38}$. The study by Juslin et al. ${ }^{38}$ revealed that MitoQ prevented apoptosis caused by oxidative damage ${ }^{38}$. Along the same lines, Adlam et $\mathrm{al}^{39}$. studied the effects of MitoQ 
in mouse models of mitochondrial oxidative damage and found decreased heart dysfunction and mitochondrial damage after ischemia reperfusion ${ }^{40,41}$. These studies suggest that MitoQ may decrease free radicals, reduce oxidative damage and prevent mitochondrial dysfunction ${ }^{31,39}$.

\section{[1.3.1.2] MitoVitE}

MitoVitE consists of the alpha-tocopherol moiety covalently attached to a TPP moiety ${ }^{7,22}$. Murphy and coworkers examined MitoVitE in cultured fibroblasts from patients with FRDA. The antioxidant was able to reach accumulation ratios of 5000-6000 units inside the mitochondrial membrane with a concentration of $1-20 \mu \mathrm{M}^{31,42}$. However, MitoVitE was found to be cytotoxic at $50 \mu \mathrm{M}^{31}$.

The investigators also found that MitoVitE was 350 times more potent than the water-soluble vitamin E, trolox. MitoVitE showed superior potency (800-fold more) than idebenone (synthetic analog of coenzyme Q10) in protecting against GSH depletion. Another important finding showed that MitoVitE prevented oxidative damage by reducing $\mathrm{H}_{2} \mathrm{O}_{2}$ caspase activity ${ }^{5,38}$. Caspases are a special class of proteases with important roles in apoptosis among other metabolic processes ${ }^{43}$.

\section{[1.3.2] SS peptides}

SS peptides are small positively charged peptides called Szeto-Schiller (SS) peptides. These peptides consist of alternating aromatic residues and basic amino $\operatorname{acids}^{5,44,45}$. In vitro experiments showed scavenging properties of tyrosine (Tyr) and dimethyltyrosine (Dmt) analogs. These aromatic-cationic peptides were able to inhibit lipid peroxidation by scavenging ROS such as $\mathrm{H}_{2} \mathrm{O}_{2}, \mathrm{HO}^{*}$ and $\mathrm{ONOO}^{-5,46}$. 
In contrast with MitoQ and MitoVitE, these peptides do not depend on the mitochondrial membrane potential to accumulate inside the inner regions of the mitochondria, even though at physiological $\mathrm{pH}$ they hold a $3+$ net charge $\mathrm{s}^{5,6,47}$. When mitochondria were depolarized using FCCP, (carbonyl cyanide 4-(trifluoromethoxy) phenylhydrazone), the percent uptake of these peptides was reduced only by $\sim 10 \%$ to $15 \%$ 5,44. Additional studies showed SS-02 (Dmt-D-Arg-Phe-Lys-NH ${ }_{2}$ ) and SS-31 (D-argDmt-Lys-Phe- $\mathrm{NH}_{2}$ ) achieved a steady state of 30 minutes, indicating a fast cellular uptake $^{5,47,48}$. The aforementioned evidence suggests that these positively charged peptides do not rely on membrane potential mechanisms. The reasons behind their ability to be selectively uptaken by the mitochondria are still unclear ${ }^{3}$.

By targeting the inner membrane, SS-31 was found to have protective activity against ischemia reperfusion injury ${ }^{5,44,49}$. The peptide SS-31 showed remarkable activity in scavenging intracellular ROS, which was reflected by its ability to inhibit mitochondrial swelling caused by calcium-induced mechanisms that mimic ischemia ${ }^{5,50}$.

\section{[1.3.3] Nitrones}

Nitrones are N-oxides of imines. They have similar chemistry with that of carbonyl compounds. The alpha carbon, also called nitronyl the carbon is susceptible to nucleophilic attack because of its electropositive nature (Figure 4). Nitrones can serve as synthetic intermediates and as spin trapping agents for the detection, quantification and, in some cases, identification of free radicals. Nitrones can aid in the detection of free radicals by forming aminoxyl-based paramagnetic adducts (Figure 5). These adducts need to be stable 
enough in order to be detected using electron paramagnetic resonance spectroscopy $(\mathrm{EPR})^{51,52}$.

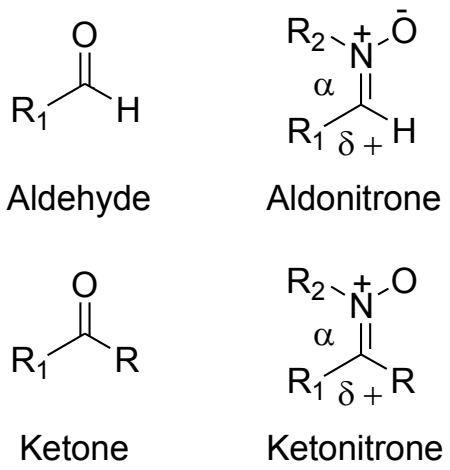

Figure 4. Electropositive carbons present on carbonyl compounds and nitrones

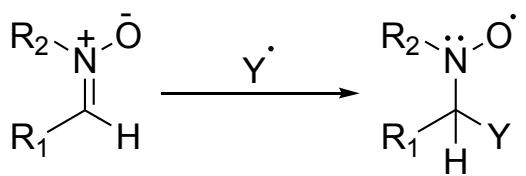

Figure 5. Generation of aminoxyl spin adduct

The EPR identification method works by measuring the magnetic moment of an unpaired electron after absorption of microwave radiation. By using the aforementioned method, the electron spin resonance (ESR) spectrum of the formed spin adduct will provide a signal due to its paramagnetic nature. The identification of the free radical may or may not be possible depending on the hyperfine splitting constant and $g$ value, which are characteristic of the type of free radical trapped ${ }^{52}$.

Nitrones have gained a lot of attention in the last decade for their role in altering cellular redox status through radical scavenging. Nitrones' scavenging activity involves trapping reactive short-lived free radicals via an addition reaction to create a more stable 
radical product, most commonly referred to as a spin adduct (Figure 6). The addition of a free radical to a nitrone results in the formation of a nitroxide. Nitroxides are stable entities because of the resonance stabilization of the unpaired electron between the nitrogen and the oxygen ${ }^{52,53}$.

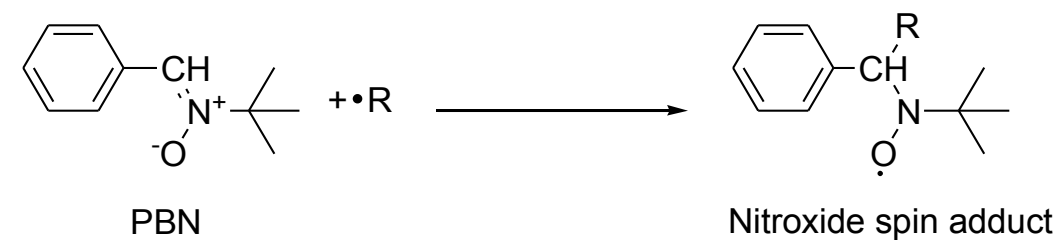

Figure 6. Spin adduct formation from PBN nitrone

As a result, the pharmacological activity of nitrones has been studied by several groups stemming from the work of Novelli et al. Investigators demonstrated the ability of PBN (alpha-phenyl-tert-butyl nitrone) to prevent death in murine models subject to LPS (lipopolysaccharide) induced shock. Thus, nitrones are good candidates to ameliorate or prevent progression of disorders involving oxidative stress due to their antioxidant nature. Mitochondriotropic nitrones will be discussed in the following sections.

\section{[1.4] Previous Work on Nitrones}

Application of nitrones as pharmacological agents have motivated research groups to synthesize compounds that could be utilized for the treatment of diseases associated with oxidative stress.

Alpha-phenyl-tert-butyl nitrone, PBN, is probably the most studied nitrone up to date because of its ability to confer neuro- protection in animal models of stroke ${ }^{54}$. Thus, several congeners such as NXY-059 and S-PBN have been synthesized (Figure 7). Despite 
the success of these phenyl nitrone derivatives in capturing ROS, they are highly hydrophilic which limit their ability to cross membranes such as the blood brain barrier. In fact, it is surmised that this was one of the reasons leading to the failure of NXY-059 in phase IIIb of human clinical trials ${ }^{16,55}$.
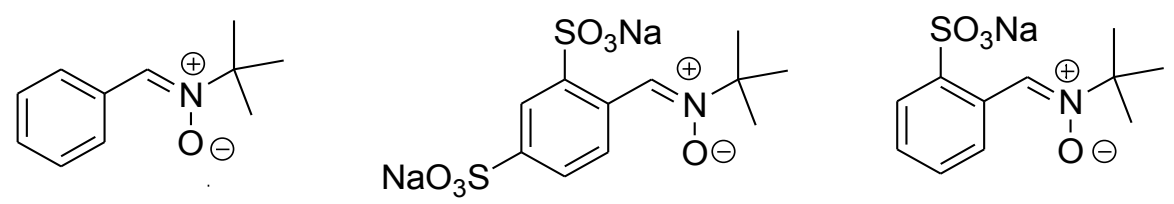

Figure 7. Nitrones: PBN, NXY-059, S-PBN

Cyclic nitrones have also been synthesized. The nitrone DMPO, 5,5-dimethyl-1pyroline $\mathrm{N}$ oxide, has demonstrated cardio-protective activity against ischemic reperfusion injury $^{52,56}$. Another major cyclic variant is DEPMPO, which has also been extensively studied for detection and sequestration of superoxide and hydroxyl radicals produced by systems in vitro and in vivo ${ }^{52}$ (Figure 8 ).
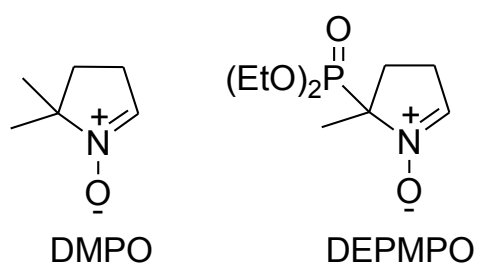

Figure 8. Cyclic nitrones

\section{[1.4.1] Mitochondriotropic Nitrones}

The laboratories of Hardy, Xu, Kalyanaraman and Murphy have synthesized nitrone compounds with the TPP cation moiety (Figure 9) ${ }^{51}$. These nitrones were synthesized with the aim of targeting the inner regions of the mitochondria. Previous 
studies have demonstrated that mitochondria-targeted antioxidants confer greater protection against oxidative damage than untargeted antioxidants ${ }^{7,57}$. However, one important limitation of these kinds of compounds is their uptake within the mitochondrial membrane $e^{5,7}$.
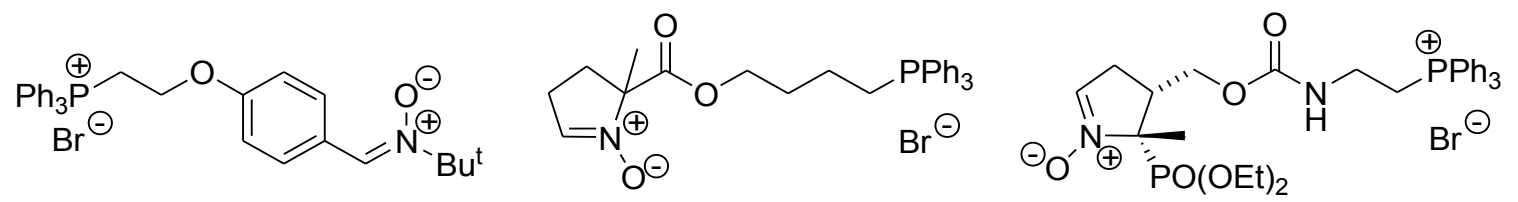

Figure 9. Structures of MitoPBN, MitoBMPO, MitoDEPMPO

Another mitochondriotropic nitrone is that prepared from a nitrone-carnitine conjugate, known as CarnDOD-7C (Figure 10). This nitrone is hypothesized to enter the mitochondria by two different mechanisms: first, it is a lipophilic cation and can accumulate in the inner membrane regions in the same manner as TPP cations; second, it could be transferred actively by carnitine acyltransferase ${ }^{51}$.

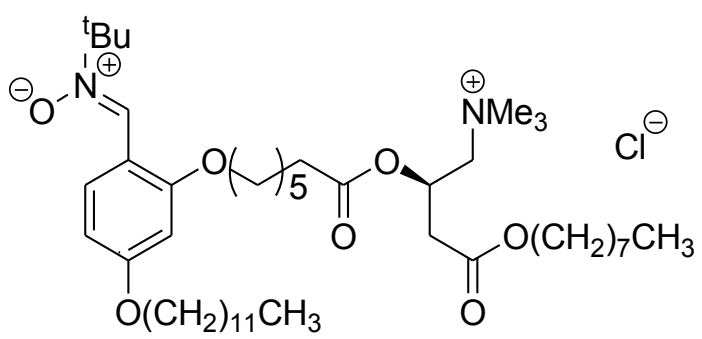

Figure 10. CarnDOD-7C nitrone

The aforementioned compounds are just a few of the many nitrones that have been synthesized. 


\section{[1.4.2] Azulenyl Nitrones}

Becker et al. first developed a new class of nitrones, named azulenyl nitrones, such as $\mathrm{AZN}^{58}$. Derivatives of AZN such as $\mathrm{W}-\mathrm{AZN}$ and STAZN were found to have oxidation potenials of 0.63 and $0.33 \mathrm{~V}$ respectively ${ }^{58-60}$ (Figure 11 ).

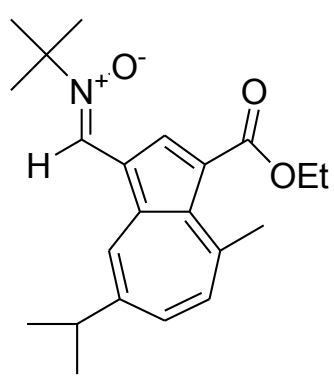

AZN

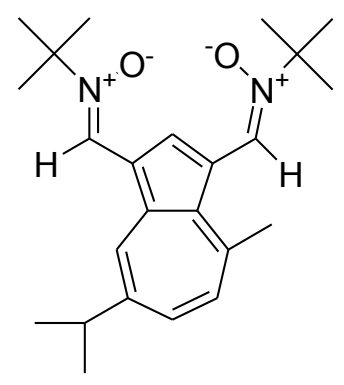

W-AZN

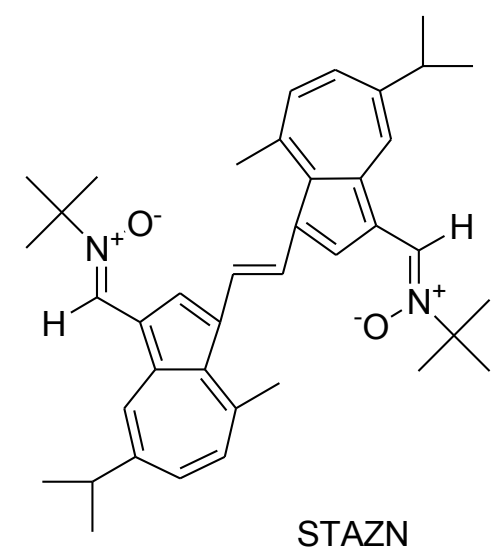

STAZN

Figure 11. Azulenyl nitrones

The lower oxidation potential of these compounds make them promising therapeutic candidates in free radial-mediated pathological conditions. Nitrones possessing lower oxidation potential is of paramount importance because STAZN, for instance, demonstrated antioxidant properties at the level of $\alpha$-tocopherol, which is an excellent antioxidant protecting cells from oxidative damage ${ }^{61,62}$. In addition, pharmacological studies of STAZN showed that it is 300 times more potent than NYX-059 at inhibiting free radical induced peroxidation in animal models of cerebral ischemia ${ }^{55,62}$. The nitrone was also able to cross the blood brain barrier as well as having a long circulating half-life in mouse models ${ }^{55}$. Thus, all these pharmacological activities indicate that STAZN and their derivatives have a promising future as potential neuroprotective agents. 
Becker's group has also synthesized a new generation of compounds called pseudoazulenyl nitrones (Figure 12).

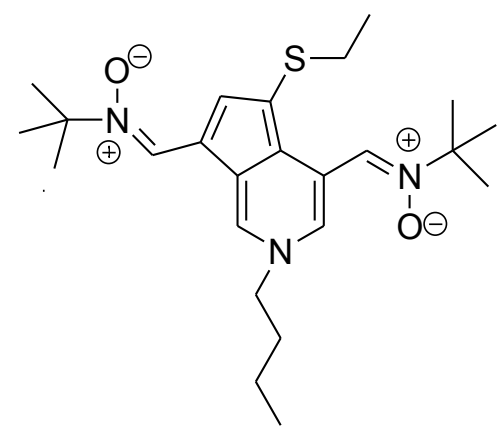

Figure 12. Pseudoazulenyl nitrone

These molecules possess a five-membered ring fused to a six-membered ring bearing an electronegative heteroatom. In contrast to azulenyl nitrones, these compounds can potentially be more efficacious in scavenging ROS since the presence of heteroatoms such as $\mathrm{O}, \mathrm{N}$ or $\mathrm{S}$ might enhance their antioxidant potency because of a decrease in oxidation potentials of these spin traps ${ }^{59,62,63}$.

Equally important, researchers in Becker's lab have also been able to synthesize novel silylated azulenyl nitrone compounds that could serve as chromotropic detection probes for superoxide radical anion ${ }^{64}$.

\section{[1.4.3] Steroidal Nitrones}

Steroidal nitrones are another promising class of antioxidants for the treatment and prevention of cerebral ischemia, Parkinson's disease, amyotropic lateral sclerosis and Alzheimer's disease. The steroid backbone of these nitrones can potentially facilitate their crossing into biological membranes. In addition, steroids have also been recognized for 
their ability to reduce inflammation, which is characteristic of many neurological diseases 65.

Contelles et al. recently reported the results of studies performed with steroidal nitrones (Figure 13). Cholesteronitrone, F2, demonstrated significant increase in neuronal viability during reperfusion compared with steroidal nitrone F3. In addition, studies done on animal models treated with F2 showed a significant decrease in the apoptotic death in the CA1 region of the hippocampus ${ }^{65}$. Therefore, these findings can aid in the development of hybrid molecules by combining the steroid and nitrone motifs, which can potentially lead to effective therapeutic agents to treat conditions associated with oxidative damage.

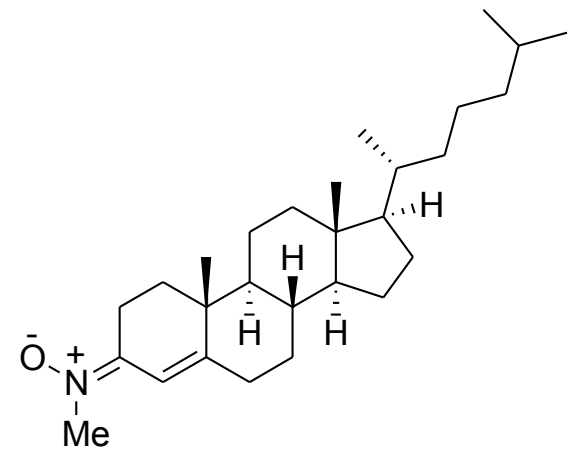

F2

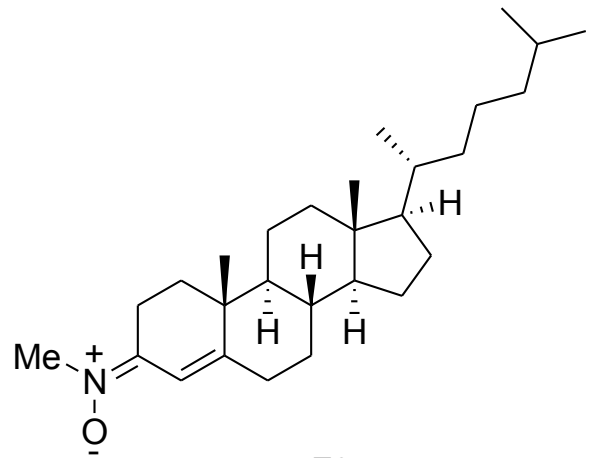

F3

Figure 13. Cholesteronitrones: F2 and F3

\section{[2] RESEARCH OBJECTIVE}

The aim of the present research is to synthesize a novel hydroxylamine of potential utility in the preparation of mitochondriotropic nitrones. The hydroxylamine, $\mathrm{N}-(3-1 H-$ imidazol-1-yl)propyl)-3-(hydroxyamino)-2,2,3-trimethylbutamide (14) is depicted on figure 14. The molecule is intended to serve as an intermediate for the synthesis of mitochondriotropic pseudoazulenyl nitrones. A novel mitochondria targeted nitrone can 
result from the condensation of this hydroxylamine with the aldehyde moiety of a pseudoazulenyl molecule (Scheme 13 in future directions section).

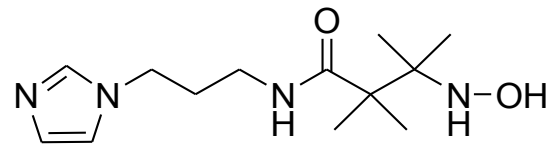

Figure 14. Hydroxylamine (14)

The hydroxylamine was carefully designed to endow it with the structural characteristics necessary to allow tethered nitrones to accumulate inside the mitochondria as well as to enable their permeation across the blood brain barrier. Among those structural considerations include: the presence of the imidazolium functionality, which provides the cationic feature needed to accumulate inside the inner regions of the mitochondria. The nitrogen in substituted congeners is expected to be protonated at physiological $\mathrm{pH}$.

Another important characteristic is the hydrocarbon backbone which contributes to an increased hydrophobic area, which is essential to permeate biological membranes including the brain. Last but not least, the presence of the amide functionality may play an interesting role for the tethered nitrone. It is hypothesized that amides (or ester linker groups) can potentially stabilize superoxide adducts through hydrogen bond interactions $^{52,66}$. Since superoxide radical is responsible for initiating the majority of free radical reactions involving oxidative stress, this added feature of amides is particularly special.

Several synthetic pathways were examined in order to make a key nitro amide precursor, $\quad \mathrm{N}$-(3-(1H-imidazol-1-yl)propyl)-2,2,3-trimethyl-3-nitrobutaminde 
necessary to attain the final hydroxylamine. The studies described in this research encompass the final synthesis of the nitro amide intermediate as well as the application of reduction methodologies using samarium diiodide, ammonium chloride and zinc. To continue this work, further reduction methods must be explored and applied to this novel intermediate to make the desired hydroxylamine.

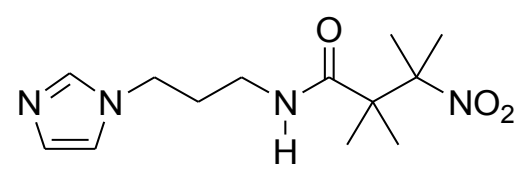

Figure 15. Nitro amide precursor (7)

\section{[3] RESULTS AND DISCUSSION}

[3.1] Direct amidation of compound (3) with hexylamine

The nitro ester intermediate, ethyl 2,2,3-trimethyl-3-nitrobutanoate (3) was synthesized according to the reported method ${ }^{67}$. The ester derivative (3) was prepared from nitration of starting material, bromoisobutyrate (1) via nucleophilic substitution to form ethyl alpha-nitroisobutyrate (2) which was reacted with the sodium salt of 2-nitropropane to form compound (3) in $81 \%$ yield as shown in scheme 1 . 


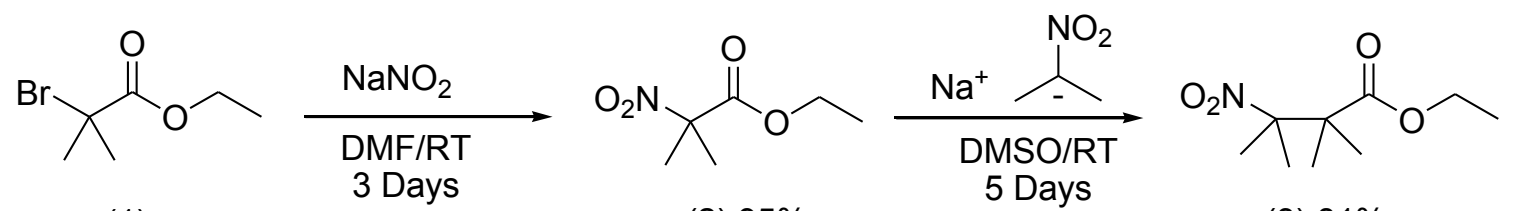

(1)

(2) $95 \%$

(3) $81 \%$

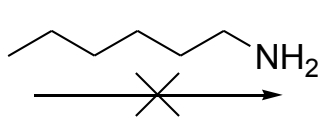

(4)

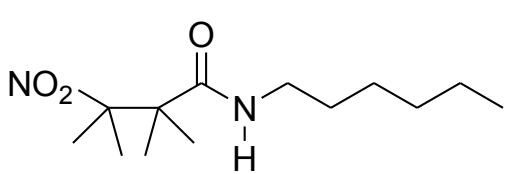

(5) Expected Product

Scheme 1 Attempted synthesis of nitro amide (5)

This model reaction was used to examine the feasibility of making amides through an acylation between the ester of interest (3) with an unhindered amine such as hexylamine (4). Two different experiments were performed. First, the amine (4) was used in excess and heated together with ester (3) at $110^{\circ} \mathrm{C}$ for 2 days. The second experimental method involved the use of excess hexylamine with ester (3) but this time higher temperature conditions were explored with a longer reaction time. The amine and ester were heated together at $154^{\circ} \mathrm{C}$ for 5 days. The experiments failed to give the expected product (5). One possible explanation of these failed reactions is that alkyl esters such as compound (3) cannot be considered activated species. On the contrary, alkyl esters most of the time are stable under usual coupling conditions. In this case our ester of interest is also very hindered with the presence of four methyl groups in the vicinity of the carbonyl carbon, which can also prevent the formation of the expected amide. As reported in the literature, alkyl esters sporadically form amides with amines under forcing conditions such as high temperatures $^{68}$. 
[3.2] Catalyzed amidation of compound (3) with imidazole-amine (6)

As discussed in the previous section, direct amidation of ester (3) was not possible using the reaction conditions specified above. Thus, another amidation attempt was made but this time using a Lewis acid catalyst, $\mathrm{MgCl}_{2}$, with the amine of interest, 1-(3aminopropyl) imidazole, (6) (Scheme 2). It was surmised that by using a Lewis acid such as $\mathrm{MgCl}_{2}$, the ester could potentially be activated by coordination of the carbonyl oxygen atom to the Lewis acid. 0.5 equivalents of $\mathrm{MgCl}_{2}$ were mixed with compound (3) and THF at room temperature. To this mixture, the imidazole- amine (6) dissolved in THF was added and left to react for 4 days. This protocol was adapted from the work described by Zhenrong et $\mathrm{al}^{69}$. Unfortunately, this amidation procedure failed to give the desired product (7). Evidence from the literature depicts successful amidation of esters by employing amine anions derived from a strong base or acid such as Grignard or alkylaluminium reagents, which does not apply to the amine used in this procedure ${ }^{69}$.

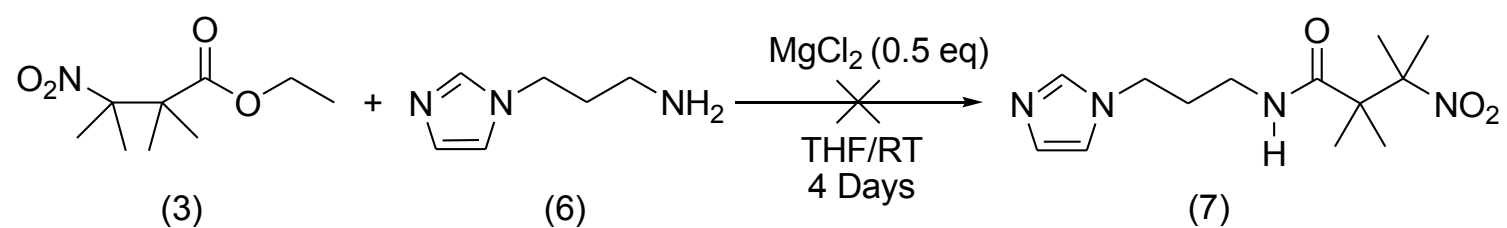

Scheme 2. Attempted synthesis of nitro amide (7) precursor using $\mathrm{MgCl}_{2}$

[3.3] Amidation of compound (8) with imidazole-amine (6)

After failure to synthesize compound (7) via amidation of esters, other attempts were made using $\alpha$-bromoisobutyryl bromide (8) as the starting material. The first synthetic pathway toward making the nitro amide intermediate (7) using this acyl bromide was to make the corresponding bromo amide, $N$-(3-1H-imidazol-1-yl)-2-bromo-2- 
methylpropanamide (9) from the imidazole-amine (6) shown in scheme 3. This bromo amide intermediate was employed to examine other potential synthetic pathways that could lead to the formation of compound (7) and ultimately the intended hydroxylamine.

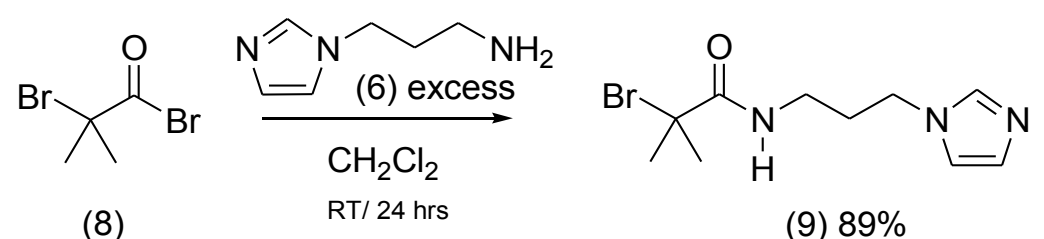

Scheme 3. Synthesis of compound (9)

The synthesis of $N$-(3-1H-imidazol-1-yl)-2-bromo-2-methylpropanamide (9) took placed smoothly and cleanly. The product was made in one step, using excess amine (6) with the aim of capturing the $\mathrm{HBr}$ formed in situ as well as having enough substrate to react with the acyl bromide. Both the amine (6) and the acyl bromide (8) were left to react for 24 hours at room temperature. After extraction of the reaction mixture with dichloromethane and water, the organic extract revealed the presence of a white solid in $89 \%$ yield. The NMR spectrum confirmed the presence of $N$-(3-1H-imidazol-1-yl)-2bromo-2-methylpropanamide (9).

As a result of the successful synthesis of compound (9), a new synthetic route was designed in order to make $N$-(3-(1H-imidazol-1-yl)propyl)-2,2,3-trimethyl-3nitrobutaminde (7) (Scheme 4). It was presumed that this one step approach could potentially afford the desired nitro amide (7) via substitution of bromine on compound (9) with the organic anion of the sodium salt of 2-nitropropane. The same mechanistic methodology was employed in the synthesis of nitro ester (3) shown in scheme 1. 


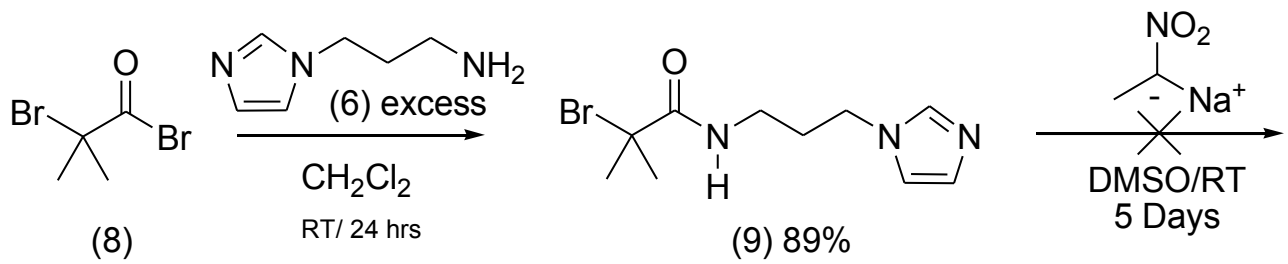<smiles>CC(C)(C(=O)NCCCn1ccnc1)C(C)(C)[N+](=O)[O-]</smiles>

(7)

Scheme 4. Attempted synthesis of nitro amide precursor through compound (9)

\section{[3.3.1] Synthesis of analog amide: Compound (10)}

To continue the attempt of synthesizing (7) employing the bromo amide (9) a new alternative route was examined. Scheme 5 shows the 4-step reaction procedure which involves the formation of another novel intermediate, $\mathrm{N}$-(3-(1H-imidazol-1-yl)propyl)-2methyl-2-nitropropanamide (10). Since direct alkylation of (9) was not possible using the sodium salt of 2-nitropropane, it was surmised that nitration of the bromo amide first and then addition of the organic salt could tentatively form the nitro amide (7).

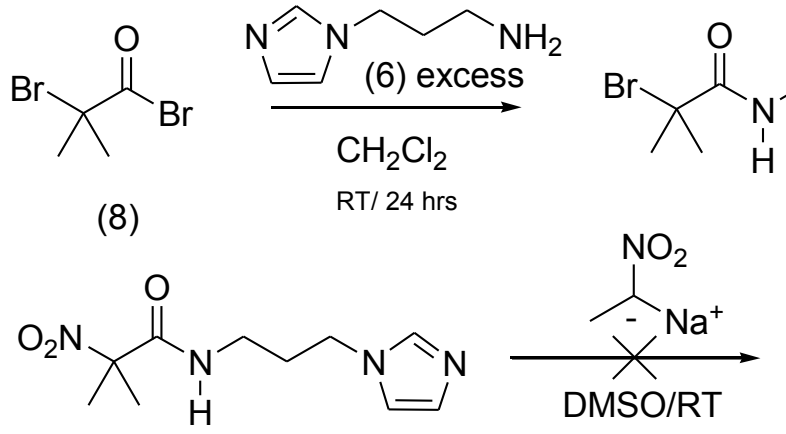

(10)
(9)<smiles>CC(C)(C(=O)NCCCn1ccnc1)C(C)(C)[N+](=O)[O-]</smiles>

(7) Expected Product

Scheme 5. Proposed synthetic approach for nitro amide precursor using compound (10) 
The nitration protocol was adapted from the work of Kornblum et al. ${ }^{70}$ with only few changes made. The reaction time used for this experiment was 72 hours instead of 2.5 hours and the extraction solvents were water and dichloromethane, unlike the solvents utilized in Kornblum's study ${ }^{70}$. The purification of (10) presented a significant challenge because of the physiochemical properties of the compounds in the reaction mixture. For example, unreacted bromo amide (9) as well as the newly formed nitro amide analog (10) were relatively polar and share solubilities in many solvents such as chloroform, dichloromethane and water to some extent. It is not surprising for these compounds to have common solubilities because of their structural similarities. In addition, removal of dimethylformamide (DMF) was very troublesome since it has a high boiling point and is slightly soluble in dichloromethane. Thus, in order to reduce DMF's content in the extracted dichloromethane layers, several extractions were made to reduce partitioning of this solvent in the dichloromethane extracts. Because the nitro amide analog (10) was synthesized as a precursor not the final product and its purification required further investigation, the impure amide (10) was further utilized to continue the synthetic approach shown in scheme 5.

The alkylation of (10) using the sodium salt of 2-nitropropane was carried out following the protocol described in the patent of Romanet's et al. ${ }^{67}$. The NMR studies did not show the presence of $N$-(3-(1H-imidazol-1-yl)propyl)-2,2,3-trimethyl-3nitrobutaminde (7). The formed product was not identified. 
[3.4] Hydrolysis of compound (3)

On the basis of the results obtained in the previous reactions, it was concluded that synthesizing the nitro amide precursor (7) would require utilization of reactive species such as acyl halides. Because synthesis of acyl halides can be accomplished through the use of carboxylic acids, the next step in the investigation involved the hydrolysis of ethyl 2,2,3trimethyl-3-nitrobutanoate (3).

\section{[3.4.1] Acid Hydrolysis}

The first attempt to hydrolyze the ester intermediate (3) involved the use of acidic conditions. Excess water and 2N hydrochloric acid ( $\mathrm{HCl})$ were added to compound (3) and the reaction mixture was heated to gentle boiling for 24 hours. The temperature of the oil bath was kept at $130{ }^{\circ} \mathrm{C}$ in order to allow escape of the azeotrope mixture of water and ethanol (bp $78.2^{\circ} \mathrm{C}$ ) while keeping the volume constant by the addition of water as the reaction proceeded. The byproducts of this reaction include the carboxylic acid, 2,2,3trimethyl-3-nitrobutanoic acid (11), and ethanol as depicted in scheme 6.

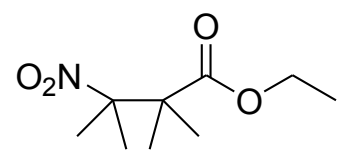

(3)

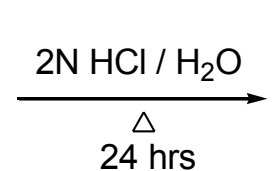

$24 \mathrm{hrs}$

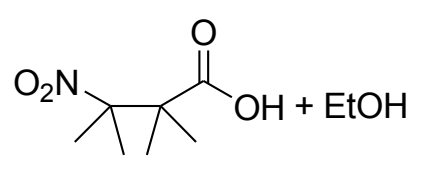

(11) $1.5 \%$

Scheme 6. Acid hydrolysis of compound (3)

After the reaction took place, extraction with diethyl ether was performed in order to remove any unreacted ester. Evaporation of the organic layer revealed a small amount of unreacted ester. As expected from the chemical structure of (11) and its polar nature, the 
acid was found to be slightly soluble in water. The aqueous extracts were placed in high vacuum conditions in order to remove water and ethanol left from the reaction.

Despite the simplicity of this hydrolysis procedure and the purity of the formed product, the yield obtained was very low $(1.5 \%)$. One tentative explanation of this poor outcome lies in the fact that acid hydrolysis of esters is a reversible process. From a mechanistic perspective, the ethanol formed with the carboxylic acid can reverse the reaction making the initial ester (3). Even though the set-up for this hydrolysis was carefully carried out to prevent condensation of ethanol back into the reaction flask, the conditions did not seem sufficient to drive the reaction towards the formation of the carboxylic acid (11). In addition, this low yield can be a sign of decarboxylation of the acid making the hydrolysis of ethyl 2,2,3-trimethyl-3-nitrobutanoate (3) via acidic conditions not a practical approach.

\section{[3.4.2] Basic Hydrolysis}

The basic hydrolysis of (3) was accomplished according to the reported method in the Romanet's patent ${ }^{67}$. The reaction consisted of two main steps. First the nitro ester (3) was mixed with methanol, sodium hydroxide, water and tetrabutylammonium sulfate. The mixture was heated at reflux for 3 days. The second step involved the acidification of the carboxylate salt, sodium 2,2,3-trimethyl-3-nitrobutanoate (12), with concentrated hydrochloric acid to form (11). The reaction scheme of this basic hydrolysis is depicted in scheme 7. 


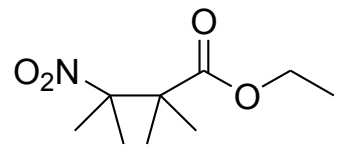

(3)

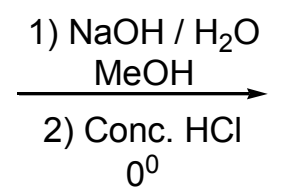

$0^{0}$

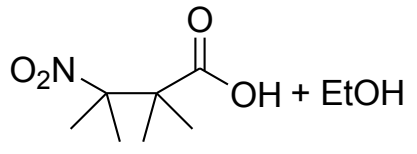

(11) $10 \%$

Scheme 7. Basic hydrolysis of compound (3)

Several procedures were performed using similar protocols. The first attempted basic hydrolysis followed the same work-up steps as shown in the patent of Romanet ${ }^{67}$. However, the product was obtained in very low yield $(\sim 5 \%)$. It was observed that during the acidification process, decarboxylation of the acid was occurring. Even though the temperature of the aqueous carboxylate mixture as well as the hydrochloric acid was kept at $0^{\circ} \mathrm{C}$ and added dropwise, constant formation of bubbles was noticeable. In addition, the nitro-acid white precipitate started to disappear completely from the acidic solution. At first, it was presumed that the nitro acid (11) was being dissolved as more acid was added to the carboxylate mixture. Nevertheless, previous solubility tests on the nitro acid (11) revealed that its solubility in water under acidic $\mathrm{pH}$ at room temperature was very low $(17 \mathrm{mg} / \mathrm{ml})$. As a result, a myriad of work-up procedural modifications were examined in order to optimize the yields of this reaction.

The most effective work-up modification consisted of complete isolation and purification of the carboxylate salt through diethyl ether washings and vacuum filtration. The organic salt (12) was completely dried and further dissolved in the minimum amount of water prior to acidification. Similar to the method reported, the carboxylate aqueous solution and the hydrochloric acid were kept cold in order to avoid decarboxylation. This small modification of protocol resulted in a significant increase in yield from $5 \%$ to $10 \%$. 
From the observations made during the acidification process, the formation of bubbles was reduced which might be an indicator that using the minimum amount of water in this step can diminish decarboxylation of the newly formed acid.

[3.5] DCC coupling of compound (11) with imidazolamine (6)

After successful hydrolysis of ethyl 2,2,3-trimethyl-3-nitrobutanoate (3), it was decided to attempt the synthesis of precursor (7) using N,N-dicyclohexyl carboiimide (DCC) with the nitro acid (11). The protocol was adapted from the work of Natsumi et $\mathrm{al}^{70}$. The synthetic approach is shown in scheme 8 .

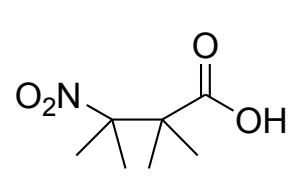

(11)

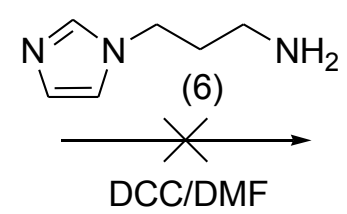

RT/One week

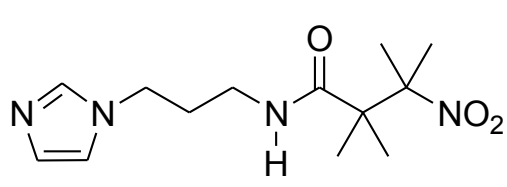

(7)

Scheme 8. Synthetic scheme of DCC catalyzed amidation of compound (11)

The mixture was reacted for one week at room temperature and the extraction was made with dichloromethane and water. NMR studies done on the dichloromethane extract failed to confirm the presence of the expected product (7). The product obtained could not be identified.

[3.6] Conversion of compound (12) to acyl chloride

As a result of the laborious task of hydrolyzing ester (3) and constant decarboxylation of the acid by both, basic and acidic methods, it was decided to move forward with the synthesis of (7) through the use of the corresponding acyl chloride, 2,2,3trimethyl-3-nitrobutanoyl chloride (13) from sodium 2,2,3-trimethyl-3-nitrobutanoate 
(12). The protocol was adapted from the patent of Romanet ${ }^{67}$ and the work of Syuji et al ${ }^{71}$. The general approach is depicted in scheme 9.

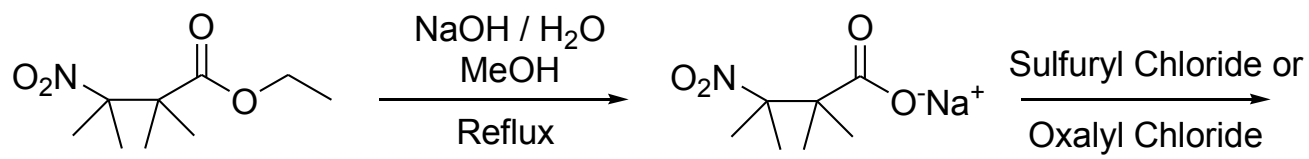

(3)<smiles>CC(C)(C(=O)Cl)C(C)(C)[N+](=O)[O-]</smiles>

(13)

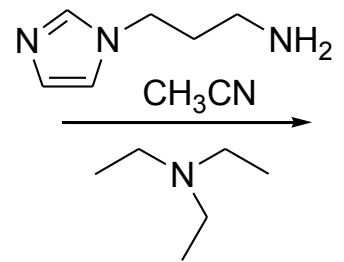

Scheme 9. General approach for acyl chloride formation using carboxylate (12)

The first attempt using (12) involved the use of sulfuryl chloride $\left(\mathrm{SO}_{2} \mathrm{Cl}_{2}\right)$ (Scheme 10). The carboxylate compound (12) was mixed with dichloromethane and $\mathrm{SO}_{2} \mathrm{Cl}_{2}$. The reaction mixture was left to react for 3 hours in a cooling bath. Subsequently, the liquid obtained, presumably the formed acyl chloride, was decanted from the reaction mixture, which contained a white solid (likely to be $\mathrm{Na}_{2} \mathrm{SO}_{4}$ ). The isolated liquid was concentrated and dissolved in acetonitrile. Then, the liquid was added dropwise to a cold mixture of amine (6) acetonitrile and triethylamine. The reaction was stirred overnight and further partitioned in water and ethyl acetate. The NMR obtained from organic extracts failed to confirm the presence of nitro amide (7). 

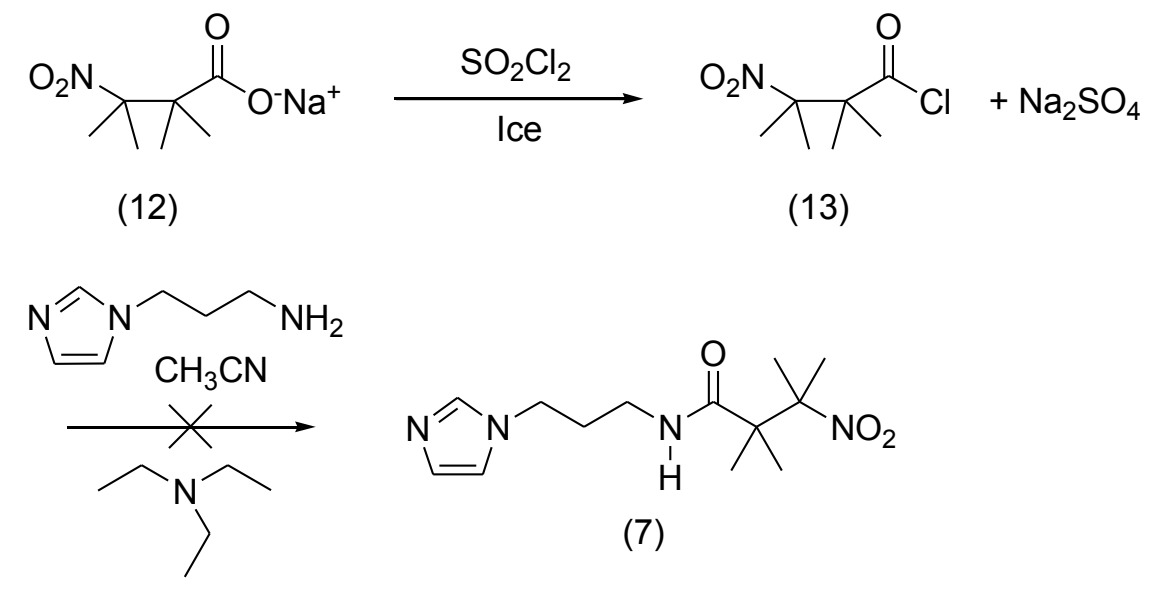

Scheme 10. Attempted synthesis of nitro amide (7) using sulfuryl chloride

Another attempt with similar protocol as described above was performed but this time the reagent was oxalyl chloride and it was added in excess (Scheme 11). NMR analyses also did not show the expected product (7). Moisture from the environment to both the newly formed acyl chloride (13) and or the sodium 2,2,3-trimethyl-3nitrobutanoate (12) could have been possible factors contributing to the failure of these reactions. In addition, the use of cooling conditions for the reaction mixture, although unlikely, could have also influenced the outcome of these reactions.
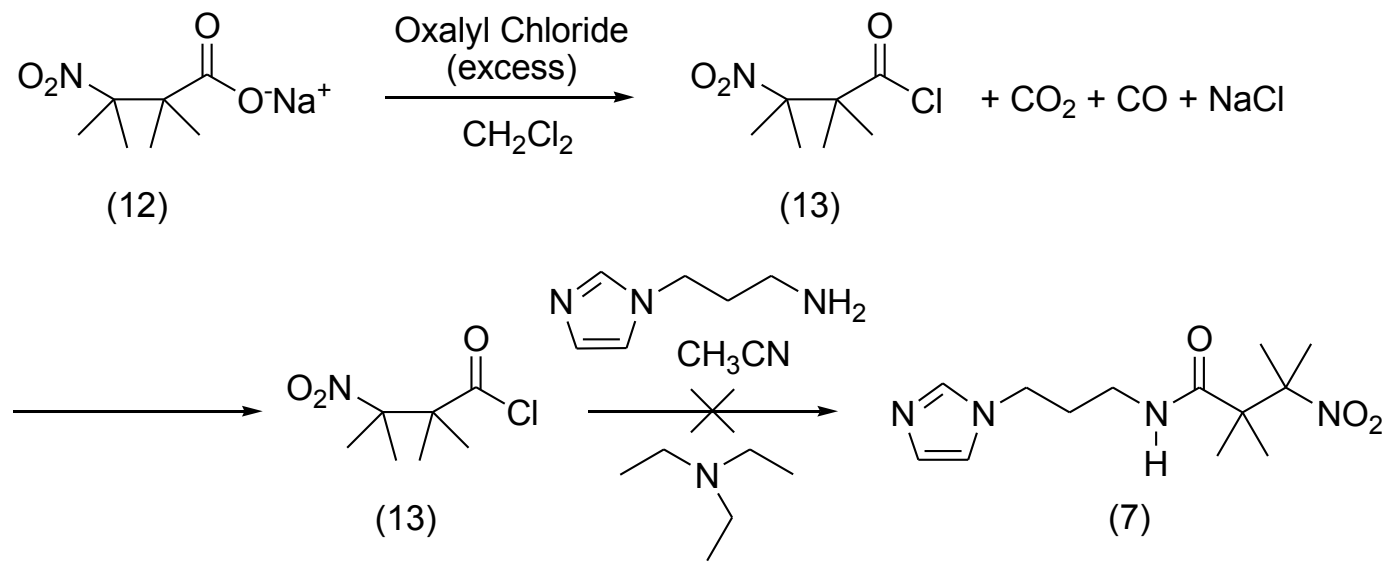

Scheme 11. Attempted synthesis of nitro amide (7) using oxalyl chloride 
[3.7] Synthesis of compound (7)

After all failed attempts to make the acyl chloride from sodium 2,2,3-trimethyl-3nitrobutanoate (12), it was decided to continue the synthesis of $N$-(3-(1H-imidazol-1yl)propyl)-2,2,3-trimethyl-3-nitrobutanamide (7) through direct conversion of 2,2,3trimethyl-3-nitrobutanoic acid (11) into 2,2,3-trimethyl-3-nitrobutanoyl chloride (13). The experimental procedure was adapted from Romanet's patent ${ }^{67}$. The complete synthetic approach is shown in scheme 12 .

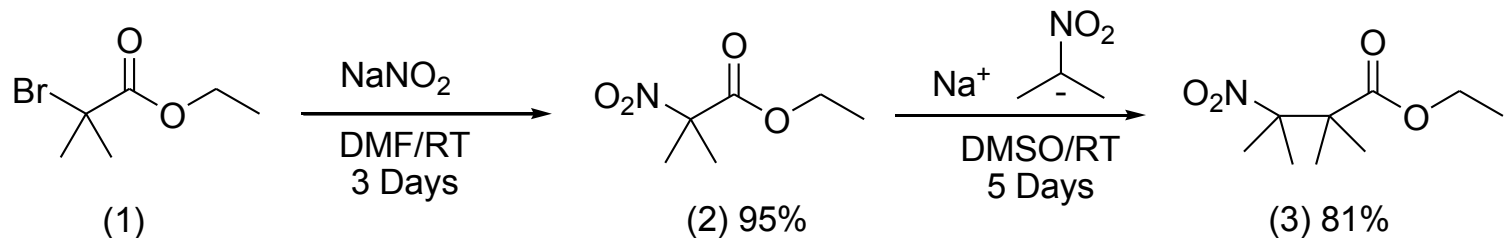

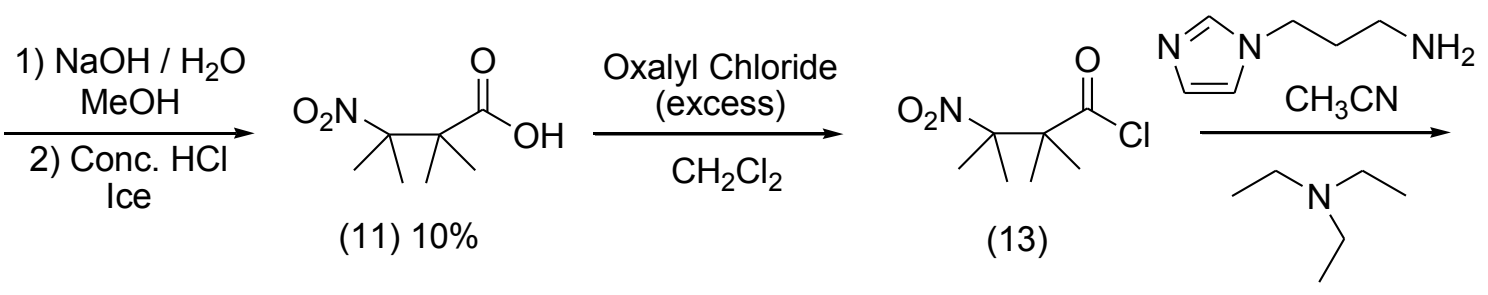<smiles>CC(C)(C)C(C)(C)C(=O)NCCCn1ccnc1</smiles>

(7) $38 \%$

Scheme 12. Synthesis of compound (7)

The nitro acid (11) was mixed with dichloromethane with a few drops of dimethylforamide and treated with oxalyl chloride. The mixture was left to react for 3 hours at room temperature and concentrated. Subsequently, the acyl chloride dissolved in acetonitrile was added dropwise to the amine mixture of acetonitrile and triethylamine. The 
reaction was stirred and allowed to react for 1 day. The reaction mixture was concentrated and partitioned in ethyl acetate and water. The ethyl acetate extracts gave the expected compound, $N$-(3-(1H-imidazol-1-yl)propyl)-2,2,3-trimethyl-3-nitrobutanamide (7) with $26 \%$ yield.

In order to optimize the yield of the reaction, several reactions conditions were examined. One of the attempted trials, consisted of adding excess oxalyl chloride to the amine mixture since it could be removed by vacuum stripping. It was surmised that insufficient amount of oxalyl chloride could greatly influence the percent yield of the formed product. As expected, by adding excess oxalyl chloride, the percent yield was increased by almost $12 \%$. In this instance, the formed nitro amide (7) was obtained in $38 \%$ yield.

\section{[3.8] Reduction of compound (7)}

After successful synthesis of the nitro amide precursor (7), two different reduction methodologies: samarium diiodide $\left(\mathrm{SmI}_{2}\right)$ or zinc with ammonium chloride were explored in order to attain the desired hydroxylamine (14). The two methods will be discussed in the following sessions:

\section{[3.8.1] Reduction with samarium diiodide}

Following the reported method of Kende et al. ${ }^{72}$, a solution of $19.53 \mathrm{mg}(0.07 \mathrm{mmol})$ of nitro amide (7) with a 2:1 mixture of $\mathrm{THF} / \mathrm{MeOH}(0.42 \mathrm{ml})$ was added to a freshly prepared mixture of $112 \mathrm{mg}$ of $\mathrm{SmI}_{2}(0.28 \mathrm{mmol})$ with $2 \mathrm{ml}$ of THF. The reaction mixture was stirred and left to react for different periods of time. The reaction was subsequently poured into a $10 \%$ solution of $\mathrm{Na}_{2} \mathrm{~S}_{2} \mathrm{O}_{3}(2 \mathrm{ml})$ and extracted several times with EtOAc. 
Using the same molar equivalents of $\mathrm{SmI}_{2}$ described above and room temperature conditions, different reaction times were examined. The reductions were carried out for: 3 and 5 minutes, 1, 8 and 24 hours. Despite the longer exposure of nitro-amide (7) to $\mathrm{SmI}_{2}$, C-13 NMR studies performed on these experiments did not show the presence of a new carbon bearing a hydroxylamino functionality, which was expected to appear around 60 ppm. All carbon-13 spectra of these experiments including the spectrum of the 24-hour reaction experiment displayed the carbon bearing the nitro group at approximately $93 \mathrm{ppm}$. Also, no signs of decomposition appeared on these experiments. Even though, the desired hydroxylamine (14) could not be obtained using the aforementioned protocol, it is suggested that further studies with samarium diiodide should be explored. Literature precedents report good yields of hydroxylamines using this method. It is surmised that perhaps employing temperatures higher than $25^{\circ} \mathrm{C}$ and/or exploring several reaction times will result in the efficient formation of the desired hydroxylamine.

[3.8.2] Reduction with ammonium chloride and zinc powder

Another reduction method utilized with the aim to obtain hydroxylamine (14) involved the use of zinc powder and ammonium chloride as reducing agents. The protocol was adapted from the work of Mitsuru et al. ${ }^{73}$ Although $12 \mathrm{mg}$ of a white solid was ultimately obtained via this procedure, C-13 NMR spectroscopy revealed that the imidazole ring was destroyed and the product could not be identified.

\section{[4] FUTURE DIRECTIONS}

To continue this work, further reduction methods need to be examined with nitro amide (7). It is recommended to use samarium diiodide employing temperatures higher 
than $25^{\circ} \mathrm{C}$ and/or exploring several reaction times in order to attain the desired hydroxylamine (14). Other protocols to convert nitro compounds to hydroxylamines shoud be examined such as those contained in the Ph.D thesis of Sacher in his synthetic studies toward lycopladine $\mathrm{H}$ under the supervision of professor Steven Weinreb at Pennsylvania State University ${ }^{74}{ }^{1}$

Once this reduction step has been successfully accomplished, the hydroxylamine (14) will be coupled with pseudoazulenyl nitrone (15) via a condensation reaction. It is surmised that by combining both molecules together, we can create a new class of pseudoazulenyl mitochondriotropic nitrones (Scheme 13).

By tethering the electron-rich nitrone (15) to the imidazole backbone, it is presumed that the final compound (17) will not only portray excellent antioxidant activity but also will be able to accumulate inside the inner regions of the mitochondria. Previous studies on azulenyl nitrones and their derivatives have shown superb antioxidant activity ${ }^{62,75}$. It is hypothesized that nitrone (15), as a congener of this class of nitrones, can potentially display the same or even lower oxidation potentials than STAZN due to the presence of this particular pseudoazulene core. Such pseudoazulenes exhibited lower oxidation potentials than those of the corresponding azulenoid counterparts ${ }^{61}$.

1 "Progress Toward a Total Synthesis of the Lycopodium Alkaloid Lycopladine H” Ph.D. Thesis Joshua R. Sacher 2012 Pennsylvania State University. 

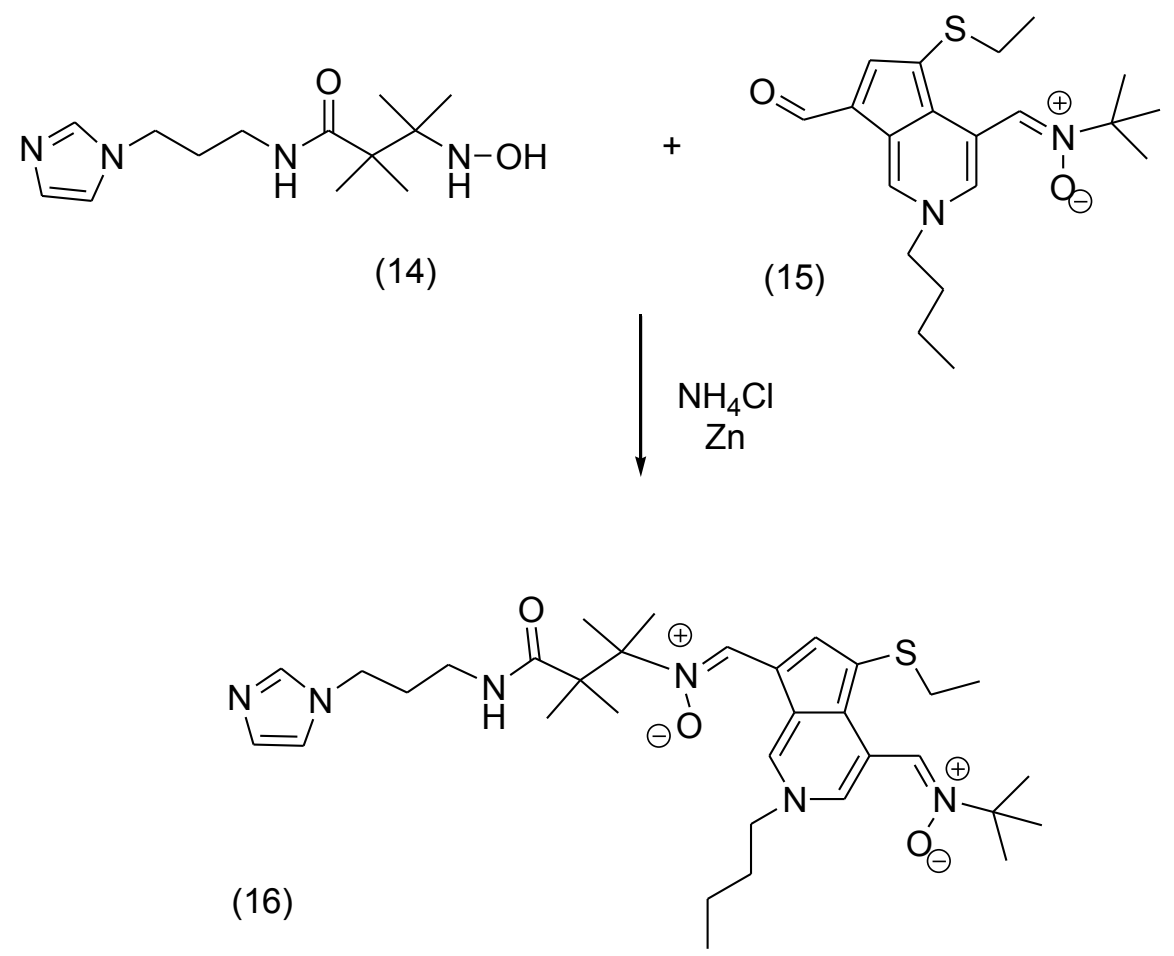<smiles>ClCc1ccccc1</smiles><smiles>CCCCn1cc(C=[N+]([O-])C(C)(C)C)c2c(SCC)cc(/C=[N+](\[O-])C(C)(C)C(C)(C)C(=O)NCCCn3cc[n+](Cc4ccccc4)c3)c-2c1</smiles>

Scheme 13. Proposed synthetic strategy for compound (17)

Another alternative approach to proceed with this investigation, will be the direct coupling of nitro amide (7) with the aldehyde moiety of nitrone (15) through the use of elemental zinc in ethanolic solution ${ }^{76}$. Although this synthetic methodology was performed 
on nitroarenes, it is a reasonable approach since the conversion of nitroalkanes to hydroxylamine can also be accomplished via the use of elemental zinc (Scheme 14)

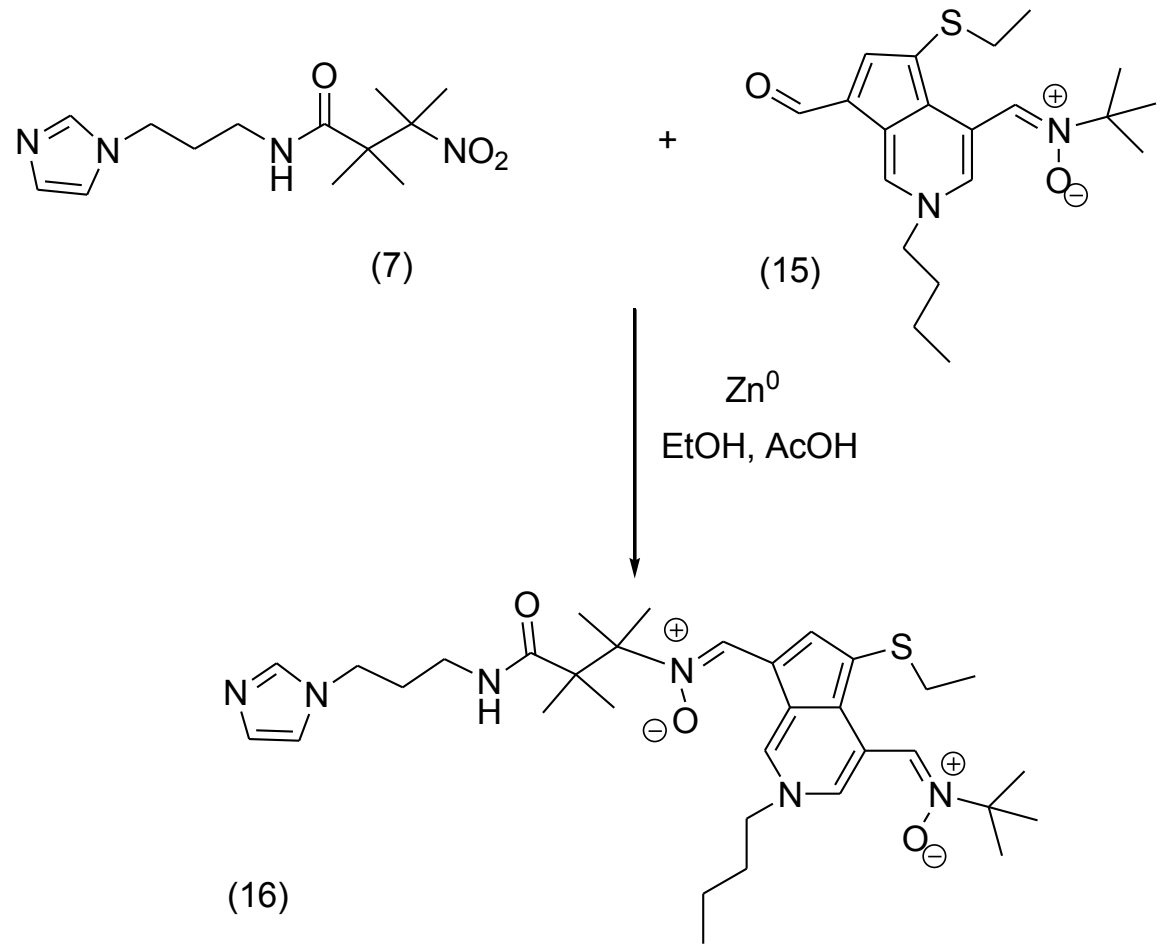

Scheme 14. Proposed synthetic strategy through direct coupling of compound (7)

Thus, nitro amide (7) can still be utilized for further synthetic studies that can potentially lead us to the development of a new class of pseudoazulenyl mitochondriotropic nitrones.

\section{[5] CONCLUSION}

Pharmacological studies have revealed important biological activity of nitrones; although the mechanism is still unclear, their inherent properties as antioxidants make them attractive candidates for therapeutic utilization in conditions associated with oxidative stress such as neurodegeneration, cancer and cardiovascular diseases ${ }^{52}$. Because many of 
these conditions involve overproduction of reactive oxygen species (ROS) and imbalances in cellular redox systems, a new generation of nitrones targeting the main site of ROS production, the mitochondria, have been studied.

Numerous synthetic methodologies were explored with the aim of synthesizing a novel hydroxylamine (14) with potential utility in the preparation of mitochodriotropic nitrones. In this quest, a novel nitro amide precursor (7) was successfully synthesized. This derivative, although not yet possessing the hydroxyl-amino functionality, warrants further reductive studies to convert it to the desired hydroxylamine, and then to the putative mitochondriotropic pseudoazulenyl nitrones.

Thus, future work on this intermediate will be pursued. Besides trying other reduction methodologies, it is also suggested to study this precursor for the direct one-pot reductive synthesis of new mitocondriotropic nitrones. Recent literature containes a report of a unique synthetic methodology describing the synthesis of alpha-aryl-N-nitrones by direct coupling of aryl aldehydes with nitro arenes through the use of elemental zinc in ethanolic solution ${ }^{76}$. With this in mind, it is highly recommended to examine this approach though direct coupling of (7) with the aldehyde moiety of a parent pseudoazulenyl nitrone (15) in order to create a new class of pseudoazulenyl mitochondriotropic nitrones.

\section{[6] EXPERIMENTAL SECTION}

\section{[6.1] General Methods}

All reagents were obtained from Sigma Aldrich. Solvents were purchased from various commercial suppliers such as Sigma Aldrich, Fisher Scientific, and Pharmcoaaper. Moisture sensitive reactions were carried out using argon gas to sweep the system 
and solutions were added to the reaction mixtures using a hypothermic syringe through a rubber stopple. Reactions were monitored by TLC using pre-coated silica gel aluminum plates with a fluorescent indicator. Visualization of plates was done with a UV lamp (wavelength $=254 \mathrm{~nm}$ ). All organic extracts were dried with anhydrous $\mathrm{MgSO}_{4}$ and removal of solvents was done under vacuum with a rotary evaporator.

One-dimensional ${ }^{1} \mathrm{H}$ NMR and ${ }^{13} \mathrm{C}$ NMR spectra were obtained using a Bruker Avance at a frequency of $400 \mathrm{MHz}$ at $298 \mathrm{~K}$. Chemical shifts were recorded in parts per million (ppm). High-resolution mass spectrometry spectra were obtained using an ultrOTOF instrument.

[6.2] Synthesis of ethyl alpha-nitro isobutyrate (2)

According to the method reported on Kornblum et al. ${ }^{70}$ Ethyl bromoisobutyrate (0.993 $\mathrm{g}, 5.09 \mathrm{mmol})$, was added to a mixture of $10.18 \mathrm{ml}$ dimethylformamide (DMF) and sodium nitrite $(0.743 \mathrm{~g}, 10.77 \mathrm{mmol})$. The reaction was stirred at room temperature and left to react for $3 \mathrm{~d}$. The reaction mixture was subsequently partitioned using diethyl ether and water. The organic layer was extracted with cold distilled water $(4 \times 30 \mathrm{ml})$ and the aqueous layer was extracted with cold diethyl ether $(4 \times 30 \mathrm{ml})$. The combined diethyl ether extracts were dried with anhydrous magnesium sulfate and vacuum filtrated. The magnesium sulfate was washed $(3 \times 20 \mathrm{ml})$ of diethyl ether and the filtrate was added to the combined organic extracts. The ether extracts were concentrated to yield $0.782 \mathrm{~g}(95 \%)$ of a yellow oil-like appearance nitro ester compound (2).

${ }^{1} \mathrm{H}$ NMR (400 MHz, $\left.\mathrm{CDCl}_{3}\right): \delta 4.19(\mathrm{q}, J=7.1 \mathrm{~Hz}, 2 \mathrm{H}), 1.73(\mathrm{~s}, 6 \mathrm{H}), 1.20(\mathrm{t}, J=7.1 \mathrm{~Hz}$, 3H). ${ }^{13} \mathrm{C} \mathrm{NMR}\left(400 \mathrm{MHz}, \mathrm{CDCl}_{3}\right): \delta 167.71,89.39,62.76,23.85,13.70$. 
[6.3] Synthesis of ethyl 2,2,3-trimethyl-3-nitrobutanoate (3)

Following the protocol reported on the patent Romanet's et al and Kerber ${ }^{67,77}$. The sodium salt of 2-nitropropane was initially prepared by adding 1 equivalent of sodium methoxide solution in methanol with 1 equivalent of 2-nitropropane at room temperature with constant stirring.

The sodium methoxide solution was prepared by careful addition of $2.3 \mathrm{~g}(0.10 \mathrm{~mol})$ of metallic sodium (Na) to $70 \mathrm{ml}$ of methanol anhydrous. After the solution became clear $9 \mathrm{~g}(0.10 \mathrm{~mol})$ of 2-nitropropane was added and then the solution was stripped at room temperature until it became viscous. To precipitate the salt of nitropropane $700 \mathrm{ml}$ of diethyl ether was added. The slurry was rapidly filtered and the white precipitate was washed with $200 \mathrm{ml}$ diethyl ether. The newly formed salt $(7.16 \mathrm{~g}, 63.88 \mathrm{mmol})$ was transferred to a round bottom flask and subjected to vacuum for $2 \mathrm{~d}$. To preserve the integrity of the salt it was covered and kept sealed at all times.

Once the salt of nitropropane was completely dried $2.2 \mathrm{~g}(19.63 \mathrm{mmol})$ was placed in a dry flask with a rubber stopper and a stirrer. The salt was swept with argon for $30 \mathrm{~min}$ and then $57.3 \mathrm{ml}$ of dimethylsufoxide (DMSO) was added through the stopper with a syringe. The system was swept with argon for another $30 \mathrm{~min}$ with the addition of $1.9 \mathrm{~g}$ (11.8 mmol) of ethyl alpha-nitro isobutyrate (2). The reaction mixture was stirred for $5 \mathrm{~d}$ at room temperature in the presence of a fluorescent lamp. The reaction was extracted with water and diethyl ether. The organic layer was dried with $\mathrm{MgSO}_{4}$ and concentrated to yield the expected ester (3), $1.94 \mathrm{~g}(81 \%)$ with a yellow oil-like appearance and mango smell.

${ }^{1} \mathrm{H}$ NMR (400 MHz, $\left.\mathrm{CDCl}_{3}\right): \delta 4.12$ (q, J=7.1 Hz, 2H), 1.63 (s, 6H), 1.26 (s, 6H), 
$1.23(\mathrm{t}, J=7.1 \mathrm{~Hz}, 3 \mathrm{H}) .{ }^{13} \mathrm{C}$ NMR $\left(400 \mathrm{MHz}, \mathrm{CDCl}_{3}\right): \delta 174,92.65,61.47,48.74,23.37$, $21.98,14.03$.

[6.4] Acid hydrolysis ethyl 2,2,3-trimethyl-3-nitrobutanoate (3)

Ethyl 2,2,3-trimethyl-3-nitrobutanoate (3) $1.00 \mathrm{~g} \mathrm{(4.92} \mathrm{mmol)} \mathrm{was} \mathrm{added} \mathrm{to} 50 \mathrm{ml}$ of $\mathrm{HCl} 2 \mathrm{~N}$ and heated to gentle boiling for $24 \mathrm{~h}$. The initial volume of the reaction mixture was measured in order to replace the evaporated water while keeping the total volume of water constant. The ethanol-water azeotrope mixture was allowed to escape in order to drive the reaction forward to obtain the carboxylic acid (11). After $24 \mathrm{~h}$ the reaction mixture was partitioned with water and diethyl ether. $7.2 \mathrm{mg}$ of unreacted ester (3) was found on the diethyl ether layer. The aqueous layer was subjected to high vacuum $(4 \mathrm{~mm} \mathrm{Hg}$ ) to remove the residual solvent. After complete evaporation of the aqueous layer $12 \mathrm{mg}(1.5 \%)$ of a white solid was obtained.

${ }^{1} \mathrm{H}$ NMR (400 MHz, $\left.\mathrm{D}_{2} \mathrm{O}\right): \delta 1.68$ (s, 6H), 1.23 (s, 6H). ${ }^{13} \mathrm{C}$ NMR (400 MHz, MeOD): $\delta$ $177.67,93.89,62.10,23.82,22.56$.

[6.5] Basic hydrolysis ethyl 2,2,3-trimethyl-3-nitrobutanoate (3)

Ethyl 2,2,3-trimethyl-3-nitrobutanoate (3) $5.87 \mathrm{~g}(28.88 \mathrm{mmol})$ was added to a solution containing $44 \mathrm{ml}$ of methanol $(\mathrm{MeOH}), 5.9 \mathrm{~g}(73.75 \mathrm{mmol})$ of $50 \%$ by weight of sodium hydroxide, $14.7 \mathrm{ml}$ of water and $0.58 \mathrm{~g}$ of $50 \%$ solution of tetrabutylammonium sulfate. The reaction mixture was heated at reflux for $3 \mathrm{~d}$. After the reaction time had elapsed, the reaction mixture turned orange and the presence of a white solid surrounding the walls of the reaction flask was observed. The solid was filtered and washed with diethyl ether to remove any unreacted ester. The solid obtained after dying was $2.50 \mathrm{~g}$. Then, the 
filtrate was concentrated to remove methanol and then partitioned with diethyl ether and water. $1.60 \mathrm{~g}$ of unreacted ester (3) was recovered from the organic extracts. The aqueous layer was evaporated to dryness under high vacuum conditions and $40^{\circ} \mathrm{C}$. The residual carboxylate salt found in the aqueous extract was $1.54 \mathrm{~g}$, which was added to the $2.50 \mathrm{~g}$ of carboxylate salt previously filtered.

After the combined solids were added to give a total of $4.04 \mathrm{~g}$ of dried carboxylate salt (12), $10 \mathrm{ml}$ of iced water was added. The amount of water added was minimal, strictly to cover the entire surface of the solid. Then, $6.16 \mathrm{mls}$ of concentrated $12 \mathrm{~N} \mathrm{HCl}$ were added carefully dropwise to the carboxylate iced solution. The temperature of the carboxylate salt solution was continuously monitored in order to avoid a rise in temperature. The bubbles produced upon addition of the acid were clear indicators of acid decarboxylation. $0.380 \mathrm{~g}$ $(10.34 \%)$ of nitro acid was obtained in the form of a white precipitate.

${ }^{1} \mathrm{H}$ NMR (400 MHz, D $\left.2 \mathrm{O}\right): \delta 1.68(\mathrm{~s}, 6 \mathrm{H}), 1.23(\mathrm{~s}, 6 \mathrm{H}) .{ }^{13} \mathrm{C}$ NMR (400 MHz, MeOD): $\delta$ $177.67,93.89,62.10,23.82,22.56$.

[6.6] Synthesis of $N$-(3-1H-imidazol-1-yl)-2-bromo-2-methylpropanamide (9)

To a stirring mixture of $2.59 \mathrm{~g}$ (19.97 mmol) of 1-(3-aminopropyl)imidazole (6) and $24 \mathrm{ml}$ of dichloromethane swept with argon, cold $2.29 \mathrm{~g}(9.98 \mathrm{mmol})$ of $\alpha$-bromoisobutyryl bromide was added dropwise. The mixture was kept in an ice bath for $1 \mathrm{~h}$. Subsequently, the mixture was left to react for $24 \mathrm{~h}$ at room temperature. The reaction mixture was washed with water $(4 \times 60 \mathrm{ml})$. Then, the combined aqueous extract was washed $(2 \times 50 \mathrm{ml})$ dichloromethane. The organic layer was dried with $\mathrm{MgSO}_{4}$, filtered and evaporated to yield $2.44 \mathrm{~g}(89 \%)$ of white solid. 
${ }^{1} \mathrm{H}$ NMR (400 MHz, $\left.\mathrm{CDCl}_{3}\right): \delta 7.51(\mathrm{~s}, 1 \mathrm{H}), 6.97$ (m, $\left.3 \mathrm{H}\right), 3.98$ (t, J=6.9 Hz, 2H), 3.27 (q, $J=6.3 \mathrm{~Hz}, 2 \mathrm{H}), 2.03(\mathrm{~m}, 2 \mathrm{H}), 1.93(\mathrm{~s}, 1 \mathrm{H}){ }^{13} \mathrm{C}$ NMR $\left(400 \mathrm{MHz}, \mathrm{CDCl}_{3}\right): \delta$ 172.76, 137.36, 129.93, 119.10, 62.84, 44.72, 37.79, 32.70, 31.13. HRMS (EI) $m / z$ calcd for $\mathrm{C}_{10} \mathrm{H}_{16} \mathrm{BrN}_{3} \mathrm{O}$ 274.16, found 274.05.

[6.7] Synthesis of $N$-(3-(1H-imidazol-1-yl)propyl)-2,2,3-trimethyl-3-nitrobutanamide (7) Following the reported method on Romanet et al. ${ }^{67} 0.380 \mathrm{~g}(2.17 \mathrm{mmol})$ of carboxylic acid (11), $4 \mathrm{ml}$ of dichloromethane, 1 drop of dimethylformamide and $1.5 \mathrm{ml}$ (17.72 mmol) of oxalyl chloride were mixed together and stirred for $3 \mathrm{~h}$ at room temperature. After $3 \mathrm{~h}$, the reaction mixture was concentrated under vacuum with a rotary evaporator. Then $8 \mathrm{ml}$ of acetonitrile were added to the concentrated acid chloride (13) mixture. Subsequently, the acid chloride was added dropwise to a previously made amine solution of $0.272 \mathrm{~g}$ (2.17 mmol) 1-(3-aminopropyl)imidazole, $0.219 \mathrm{~g}$ (2.17 mmol) of triethylamine and $1 \mathrm{ml}$ of acetonitrile. The reaction was stirred at room temperature for 1 d. Then, the reaction mixture was concentrated and partitioned with water and ethyl acetate. The aqueous layer was washed with ethyl acetate $(4 \times 10 \mathrm{ml})$. The organic layer was partitioned with water $(4 \times 10 \mathrm{ml})$, dried with $\mathrm{MgSO}_{4}$ and concentrated to give $0.230 \mathrm{~g}$ (37.6\%) of the expected nitro amide (7) as a white solid.

${ }^{1} \mathrm{H}$ NMR (400 MHz, CDCl $)$ ): $\delta 7.48(\mathrm{~s}, 1 \mathrm{H}), 7.0(\mathrm{~m}, 2 \mathrm{H}), 6.08(\mathrm{~s}, 1 \mathrm{H}), 3.99(\mathrm{t}, J=6.9 \mathrm{~Hz}$, 2H), 3.22 (q, J=6.2 Hz, 2H), 1.99 (m, $2 \mathrm{H}), 1.67$ (s, 6H), 1.28 (s, 6H) ${ }^{13} \mathrm{C}$ NMR (400 MHz, $\left.\mathrm{CDCl}_{3}\right): \delta 173.64,137.10,129.56,118.88,93.58,48.19,44.71,37.25,30.87,23.23,22.06$ HRMS (EI) $m / z$ calcd for $\mathrm{C}_{13} \mathrm{H}_{22} \mathrm{~N}_{4} \mathrm{O}_{3} 282.17$, found $(\mathrm{M}+\mathrm{H})^{+} 283.17$. 


\section{LIST OF APPENDICES}

NAME

PAGE

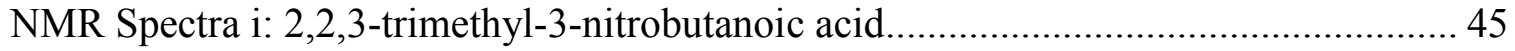

NMR Spectra ii: ethyl 2,2,3-trimethyl-3-nitrobutanoate .......................................... 46

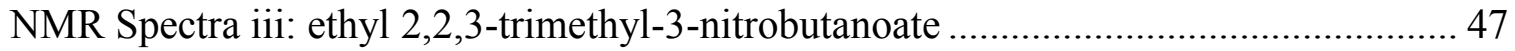

NMR Spectra iv: $N$-(3-(1H-imidazol-1-yl)propyl)-2,2,3-trimethyl-3-nitrobutanamide ... 48

NMR Spectra v: N-(3-(1H-imidazol-1-yl)propyl)-2,2,3-trimethyl-3-nitrobutanamide .... 49

NMR Spectra vi: $N$-(3-1H-imidazol-1-yl)-2-bromo-2-methylpropanamide ................... 50

NMR Spectra vii: $N$-(3-1H-imidazol-1-yl)-2-bromo-2-methylpropanamide .................. 51

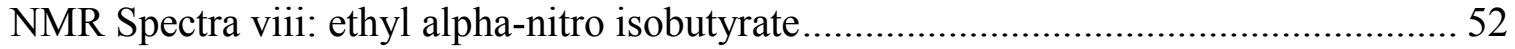

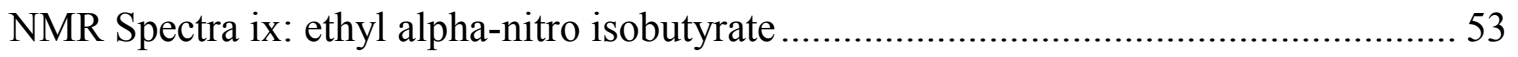

HRMS Report 1: N-(3-(1H-imidazol-1-yl)propyl)-2,2,3-trimethyl-3-nitrobutanamide...57

HRMS Report 2: N-(3-1H-imidazol-1-yl)-2-bromo-2-methylpropanamide.............63 
NMR Spectra summary

\begin{tabular}{|c|c|c|}
\hline NMR SPECTRA & NAME & STRUCTURE \\
\hline $\mathrm{i}$ & $\begin{array}{l}\text { 2,2,3-trimethyl-3- } \\
\text { nitrobutanoic acid }\end{array}$ & \\
\hline ii & $\begin{array}{l}\text { ethyl 2,2,3-trimethyl-3- } \\
\text { nitrobutanoate }\end{array}$ & \\
\hline $\begin{array}{l}\text { iv } \\
\mathrm{v}\end{array}$ & $\begin{array}{c}N \text {-(3-(1H-imidazol-1- } \\
\text { yl)propyl)-2,2,3-trimethyl- } \\
\text { 3-nitrobutanamide }\end{array}$ & $\boldsymbol{H}$ \\
\hline $\begin{array}{l}\text { vi } \\
\text { vii }\end{array}$ & $\begin{array}{c}N-(3-1 H \text {-imidazol-1-yl)-2- } \\
\text { bromo-2- } \\
\text { methylpropanamide }\end{array}$ & \\
\hline $\begin{array}{l}\text { viii } \\
\text { ix }\end{array}$ & $\begin{array}{l}\text { ethyl alpha-nitro } \\
\text { isobutyrate }\end{array}$ & \\
\hline
\end{tabular}




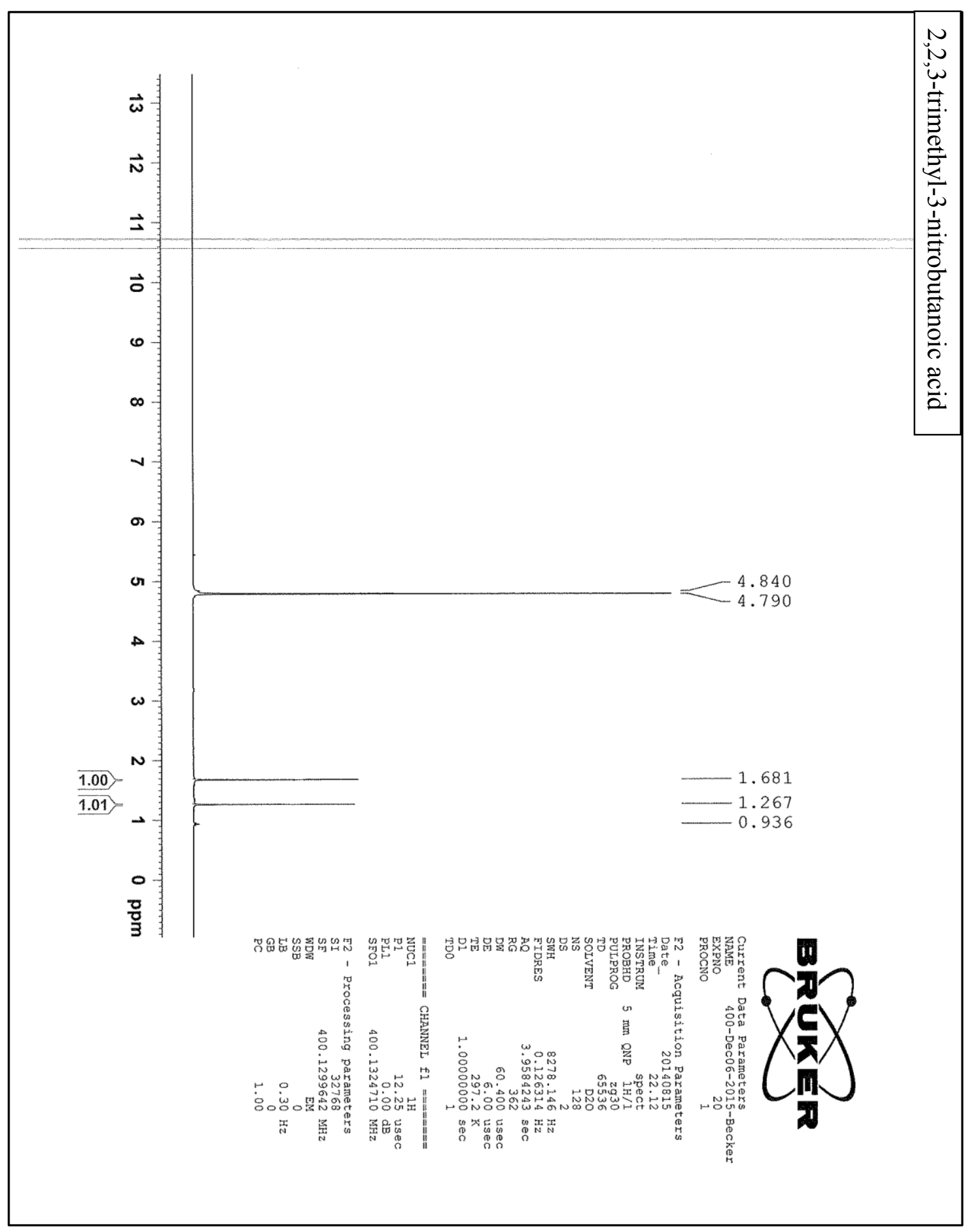

NMR Spectra i: 2,2,3-trimethyl-3-nitrobutanoic acid 


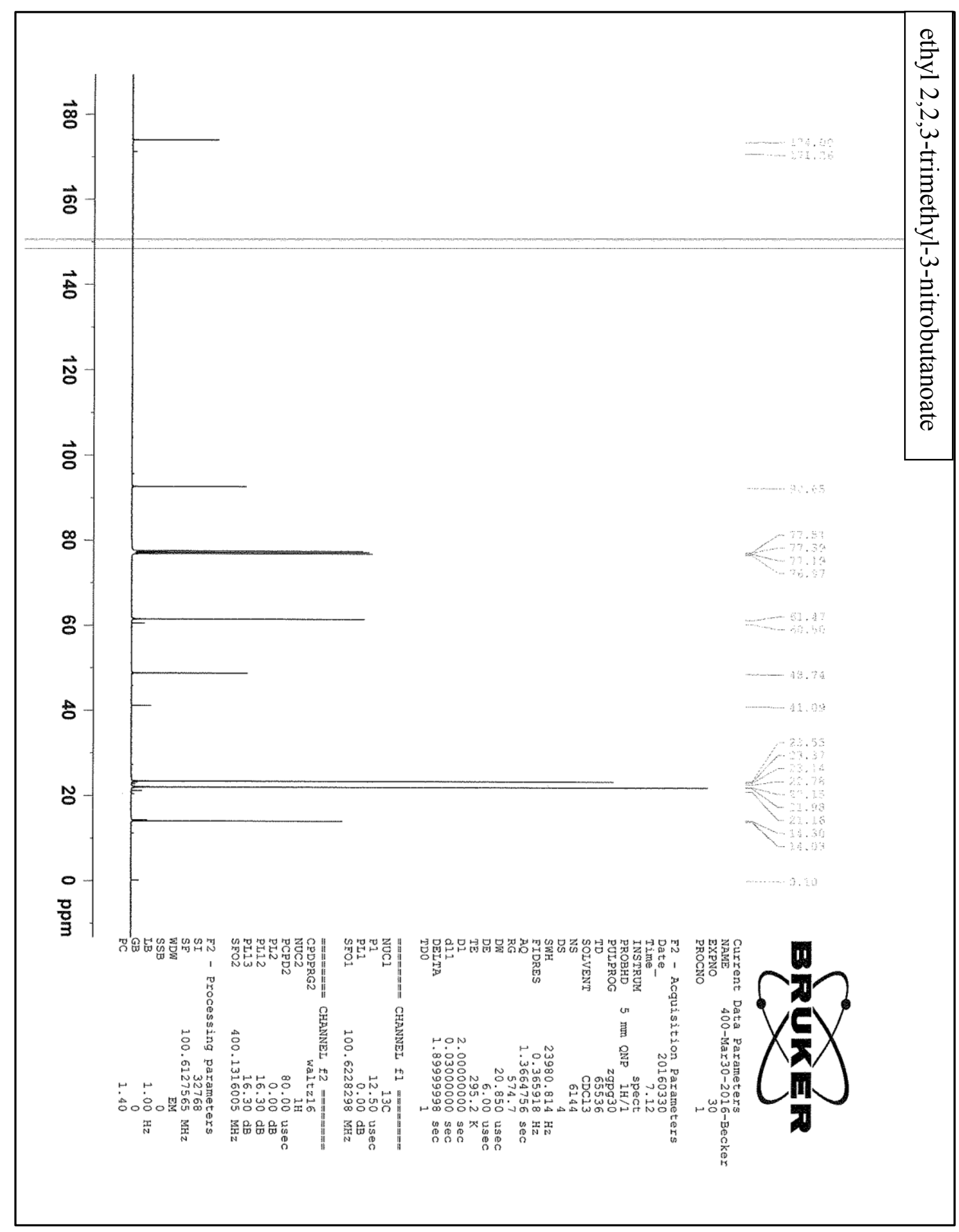

NMR Spectra ii: ethyl 2,2,3-trimethyl-3-nitrobutanoate 


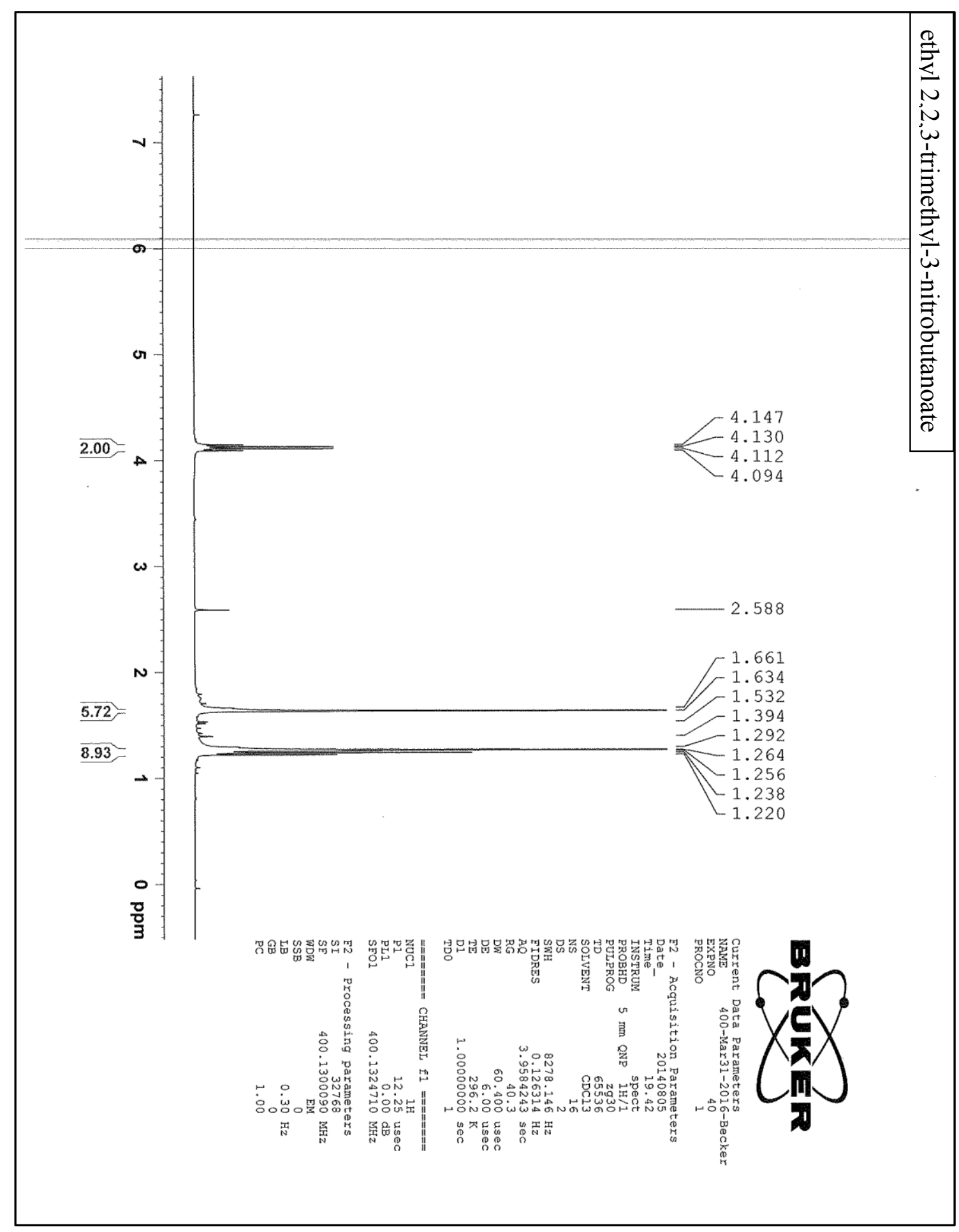

NMR Spectra iii: ethyl 2,2,3-trimethyl-3-nitrobutanoate 


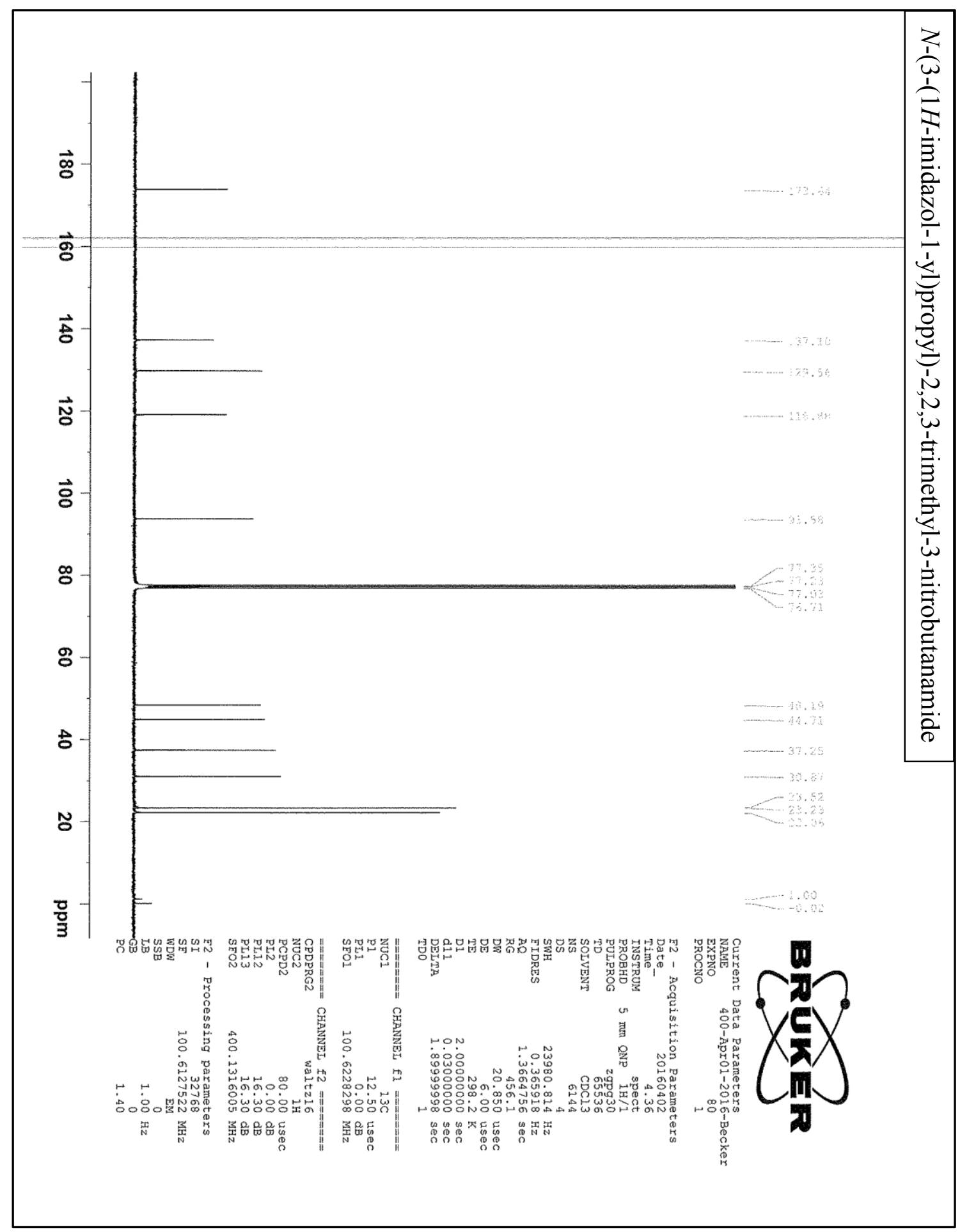

NMR Spectra iv: N-(3-(1H-imidazol-1-yl)propyl)-2,2,3-trimethyl-3-nitrobutanamide 


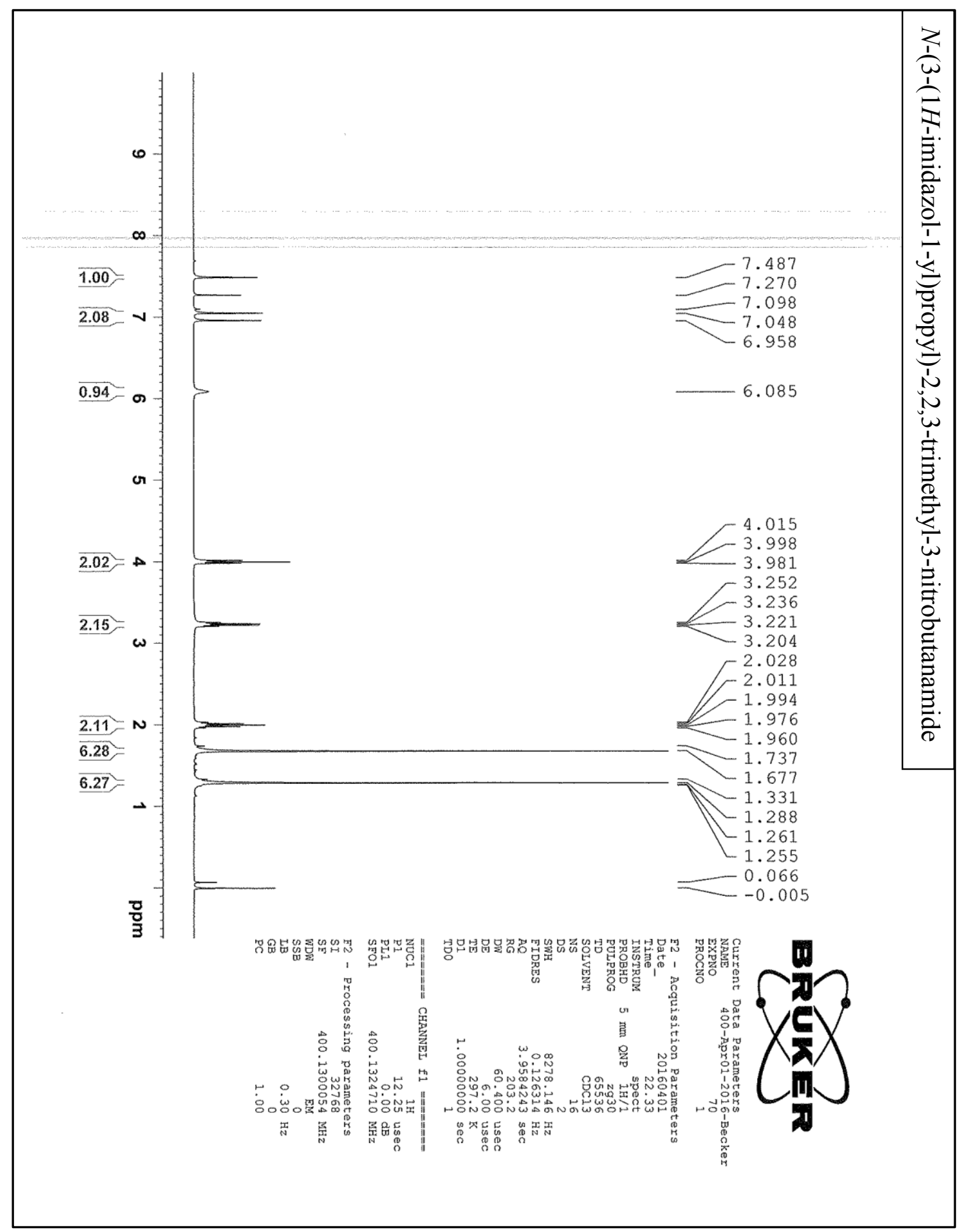

NMR Spectra v: $N$-(3-(1H-imidazol-1-yl)propyl)-2,2,3-trimethyl-3-nitrobutanamide 


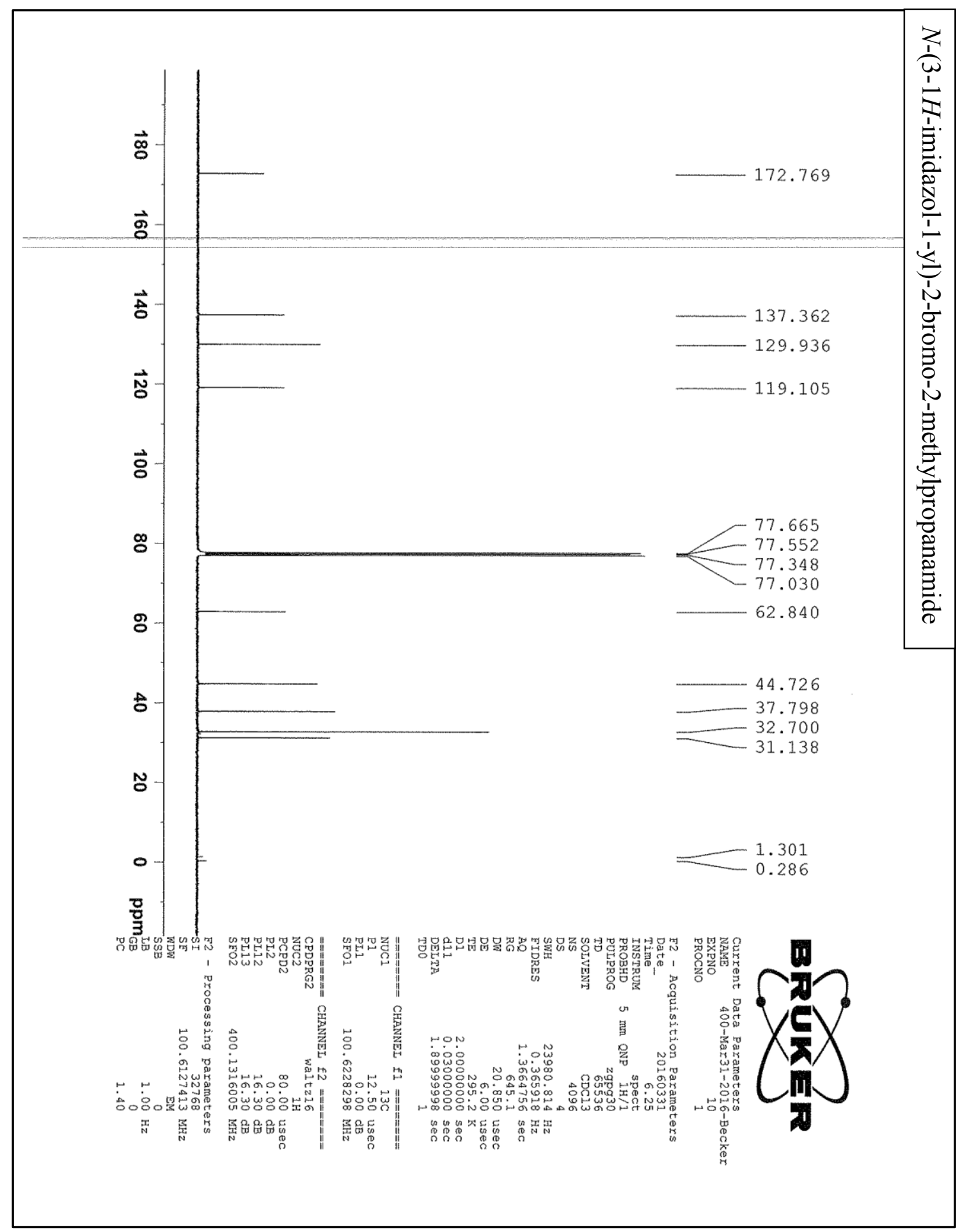

NMR Spectra vi: $N$-(3-1H-imidazol-1-yl)-2-bromo-2-methylpropanamide 


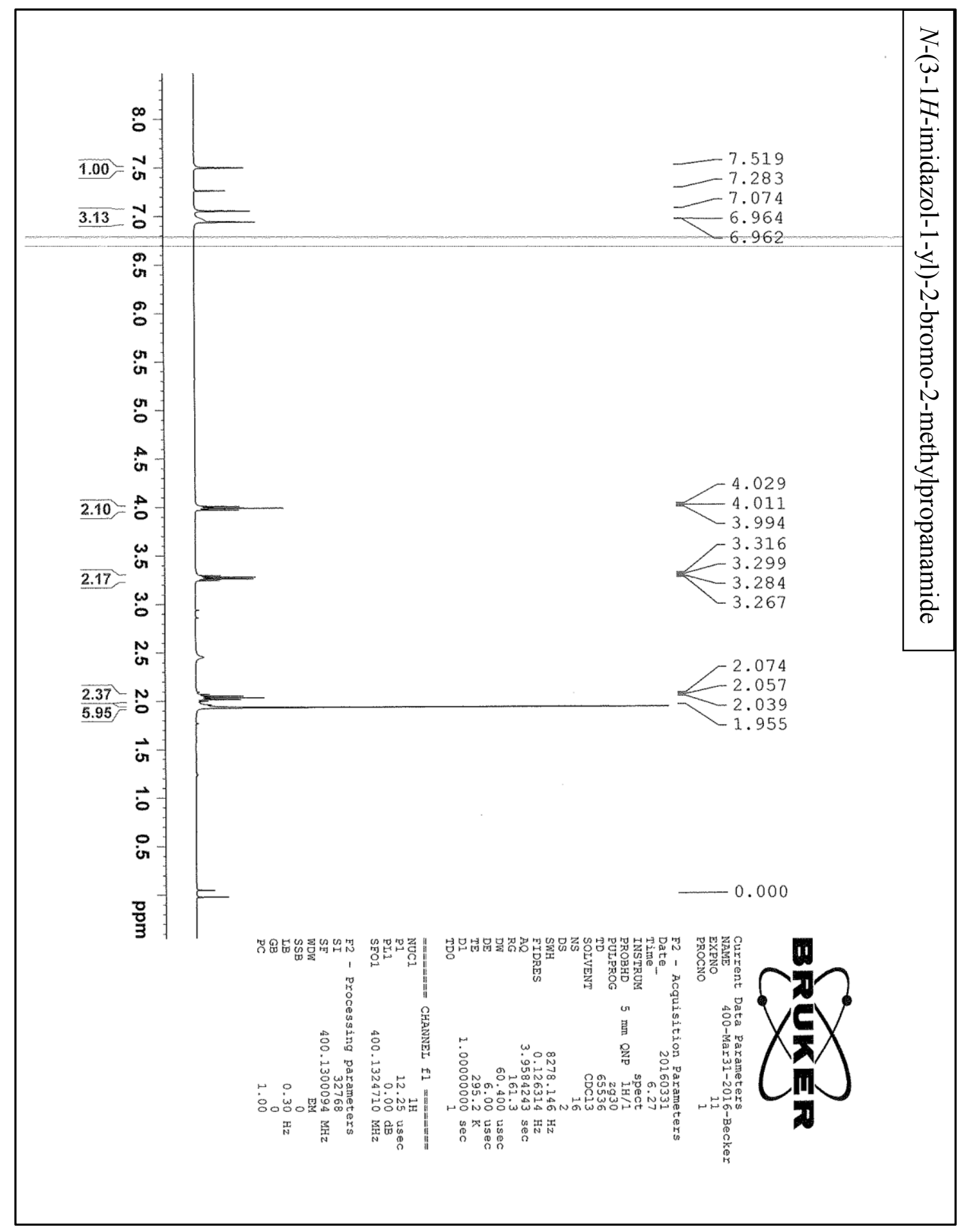

NMR Spectra vii: $N$-(3-1H-imidazol-1-yl)-2-bromo-2-methylpropanamide 


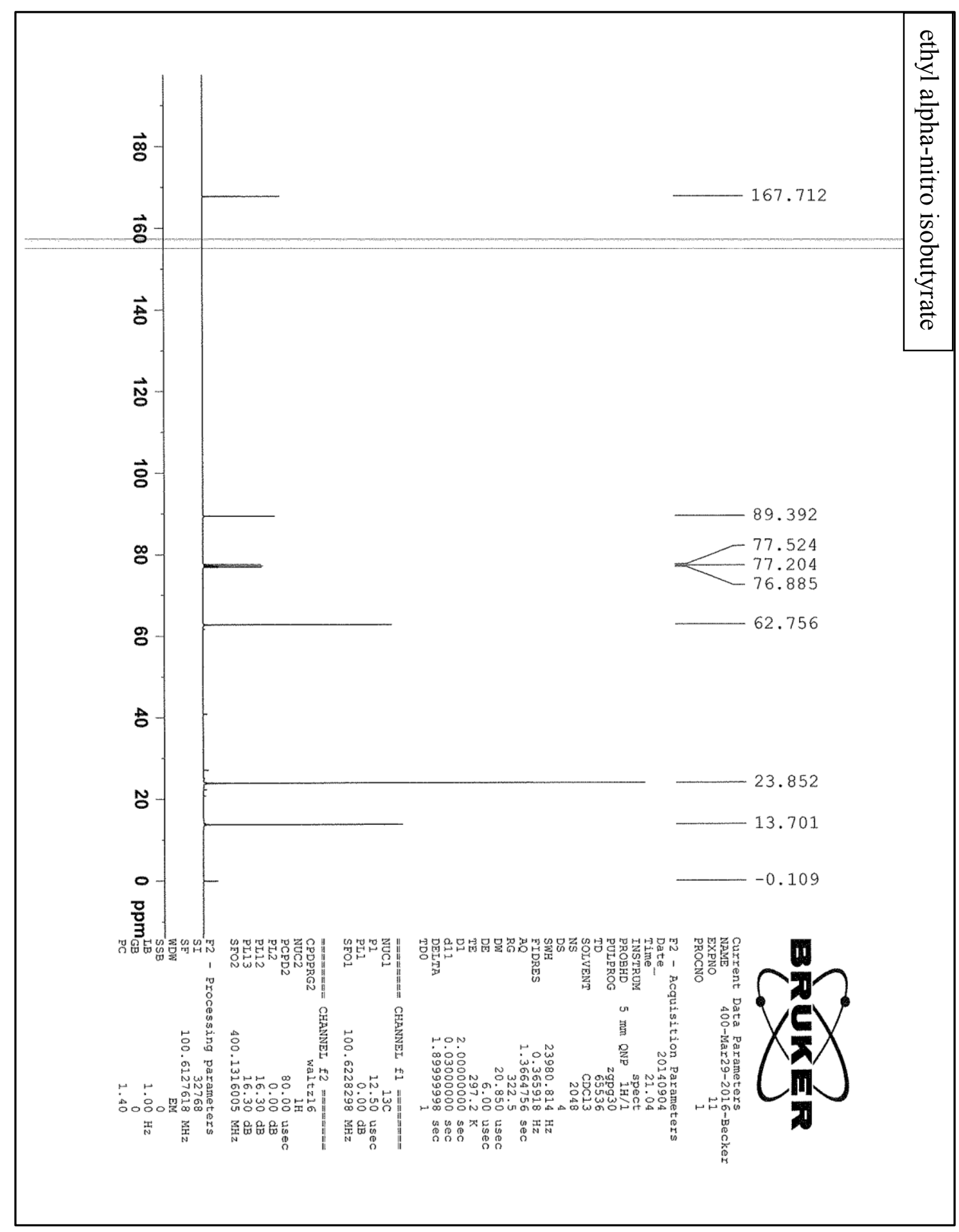

NMR Spectra viii: ethyl alpha-nitro isobutyrate 


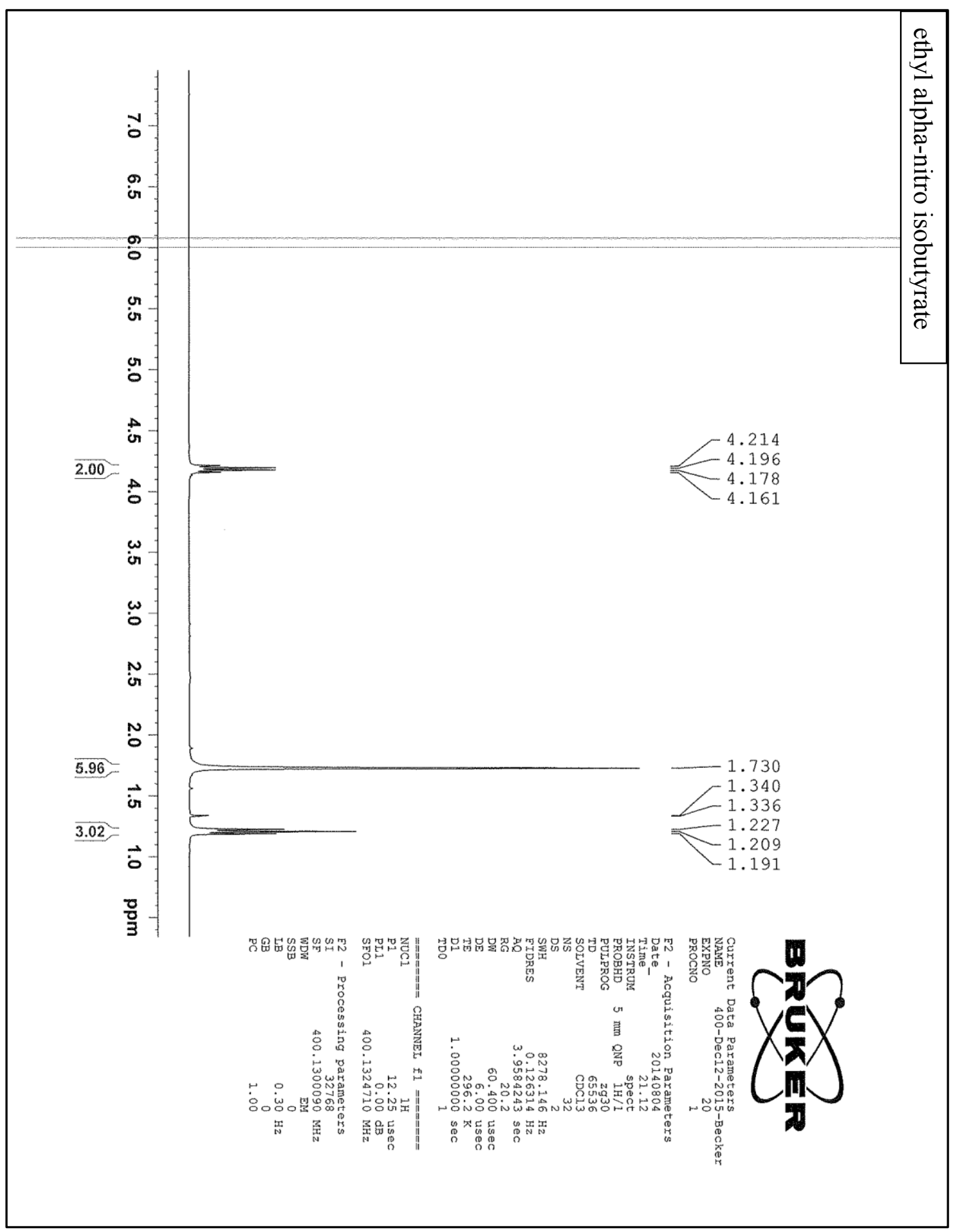

NMR Spectra ix: ethyl alpha-nitro isobutyrate 
HRMS Report 1: $N$-(3-(1H-imidazol-1-yl)propyl)-2,2,3-trimethyl-3-nitrobutanamide

\title{
111 INERARTIONAL Advanced Mass Spectrometry Facility
}

\author{
Sample Analysis Report
}

\author{
Report Date: $\quad$ April 1, 2016 \\ Request date: $\quad$ March 31, 2016 \\ Sample Received: $\quad$ April 1, 2016 \\ Request description: One sample (GP Amide) was submitted for TOF HRMS analysis by \\ Gloria Perez on 2016-4-01. \\ Report contents: \\ 1. + ESI MS Report Summary \\ 2. Submission document \\ 3. Obtained TOF +ESI HRMS spectrum Tune Mixture Blank and Sample m/z 200-1000. \\ 4. Obtained TOF +ESI HRMS spectrum Tune Mixture Blank and sample $\mathrm{m} / \mathrm{z}$ 200-310. \\ 5. Obtained TOF +ESI HRMS spectrum Tune Mixture Blank, Sample and theoretical \\ isotope pattern $[\mathrm{M}+\mathrm{H}]^{+} \mathrm{m} / \mathrm{z} 280-288$. \\ 6. Obtained TOF +ESI HRMS spectrum Tune Mixture Blank and Sample m/z 300-600 \\ 7. Obtained TOF +ESI HRMS spectrum Tune Mixture Blank and Sample m/z 600-1000.
}

Methods: The sample was mixed 1:1 with Tune Mixture and directly infused/analyzed by $(+)$ ESI mode on an ultrOTOF-Q instrument.

Report summary: An ion was observed potentially corresponding to the sample submitted at $m / z$ at $283.1779(4.9 \mathrm{ppm})$ for the $[\mathrm{M}+\mathrm{H}]^{+}$.

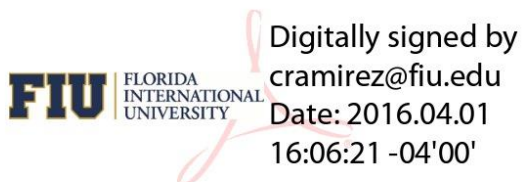

Advanced Mass Spectrometry Facility

Department of Chemistry and Biochemistry. 11200 SW $8^{\text {th }}$ ST. Room CP-174 Miami, FL. 33199.

Phone: 305.348.6656 - Fax: 305.348.3772 - E-mail: cramirez@fiu.edu 


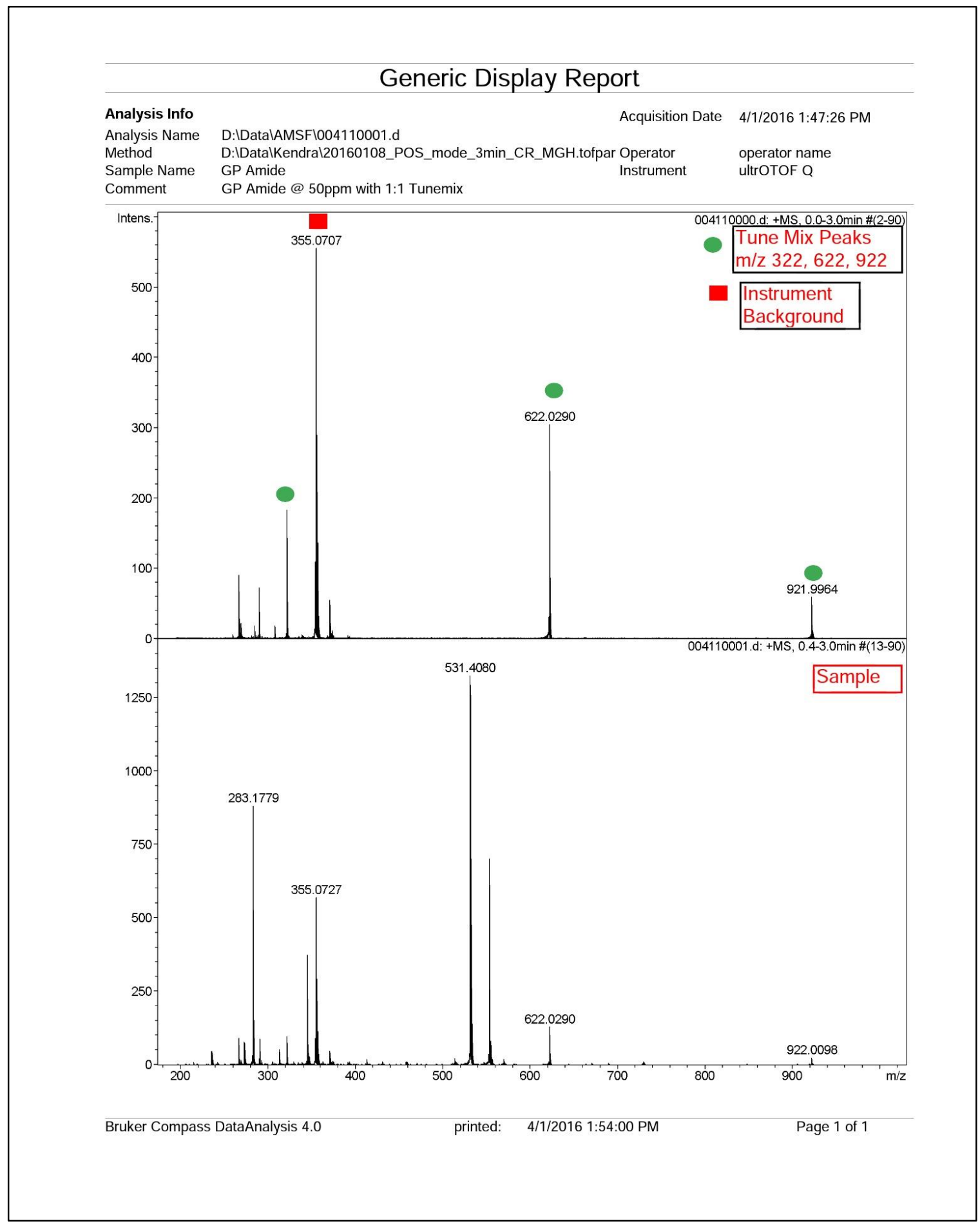


Generic Display Report

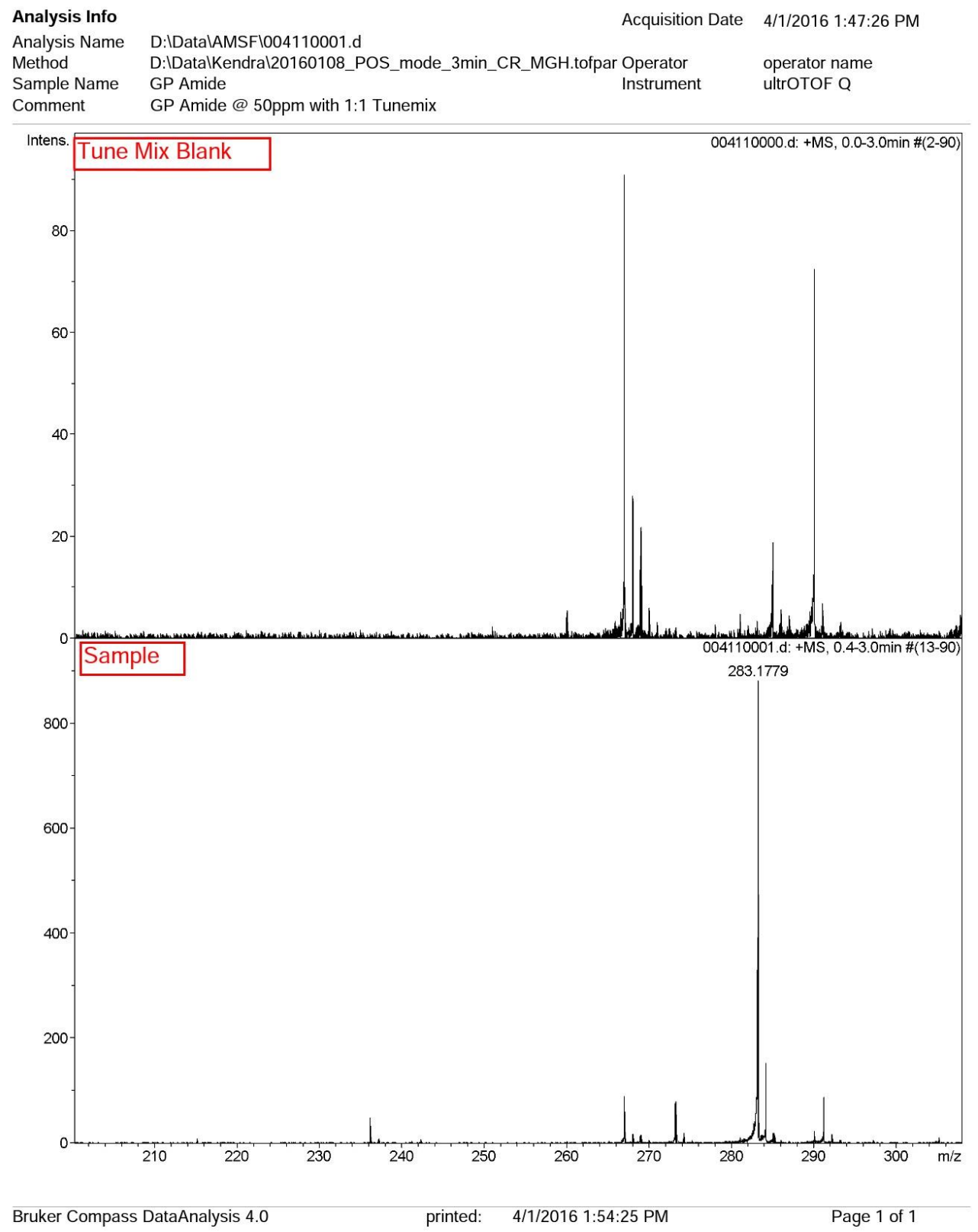




\section{Generic Display Report}

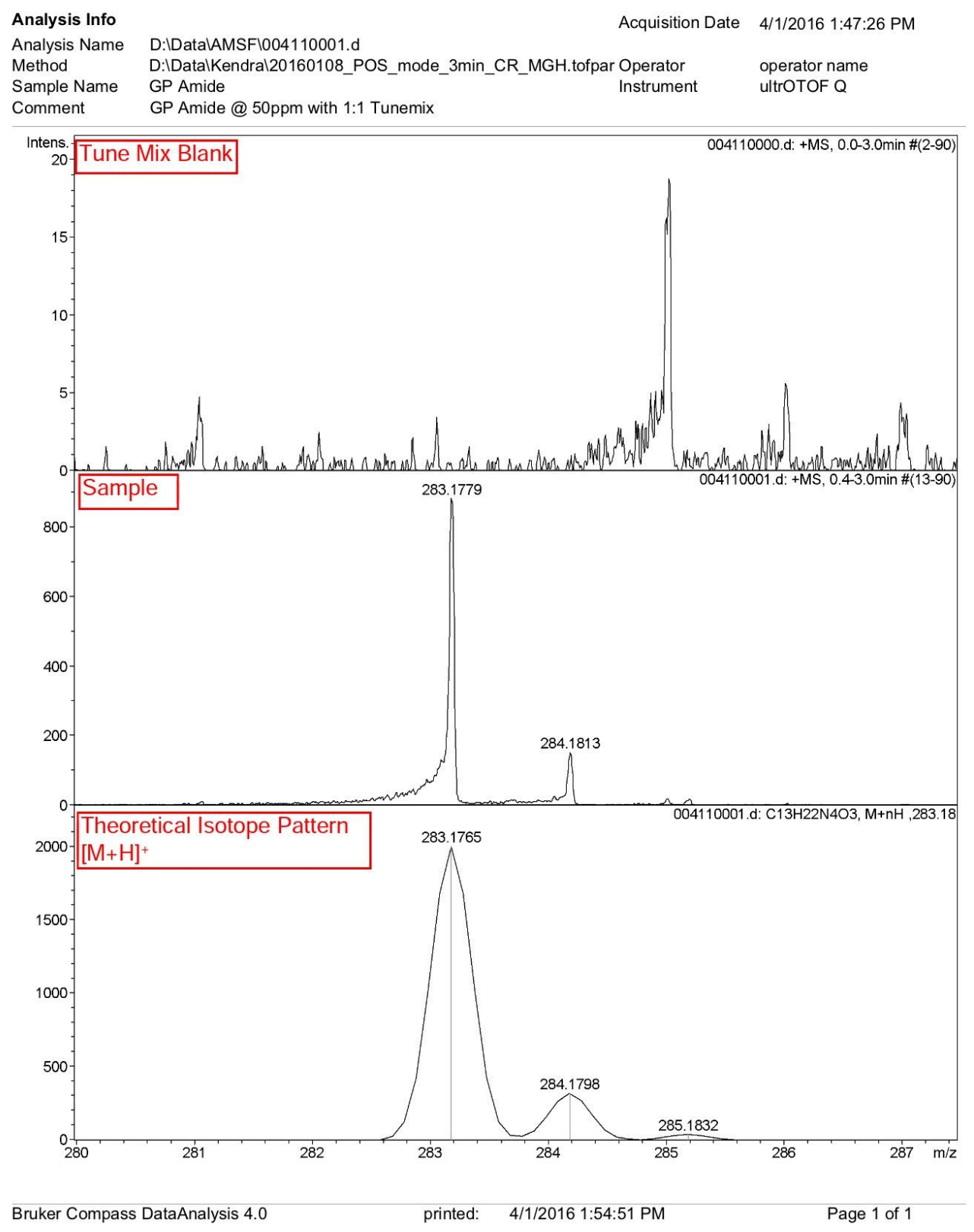




\section{Generic Display Report}

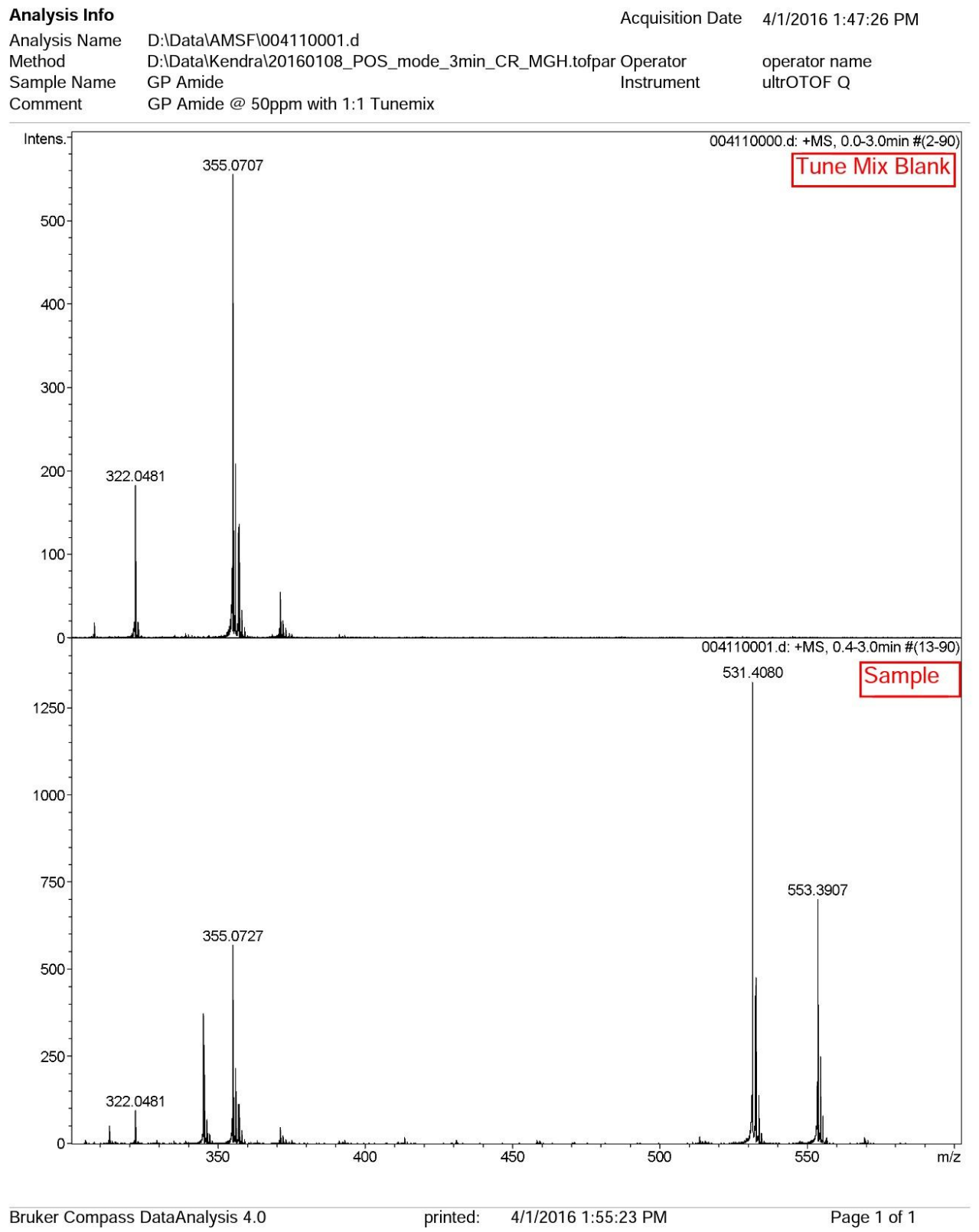




\section{Generic Display Report}

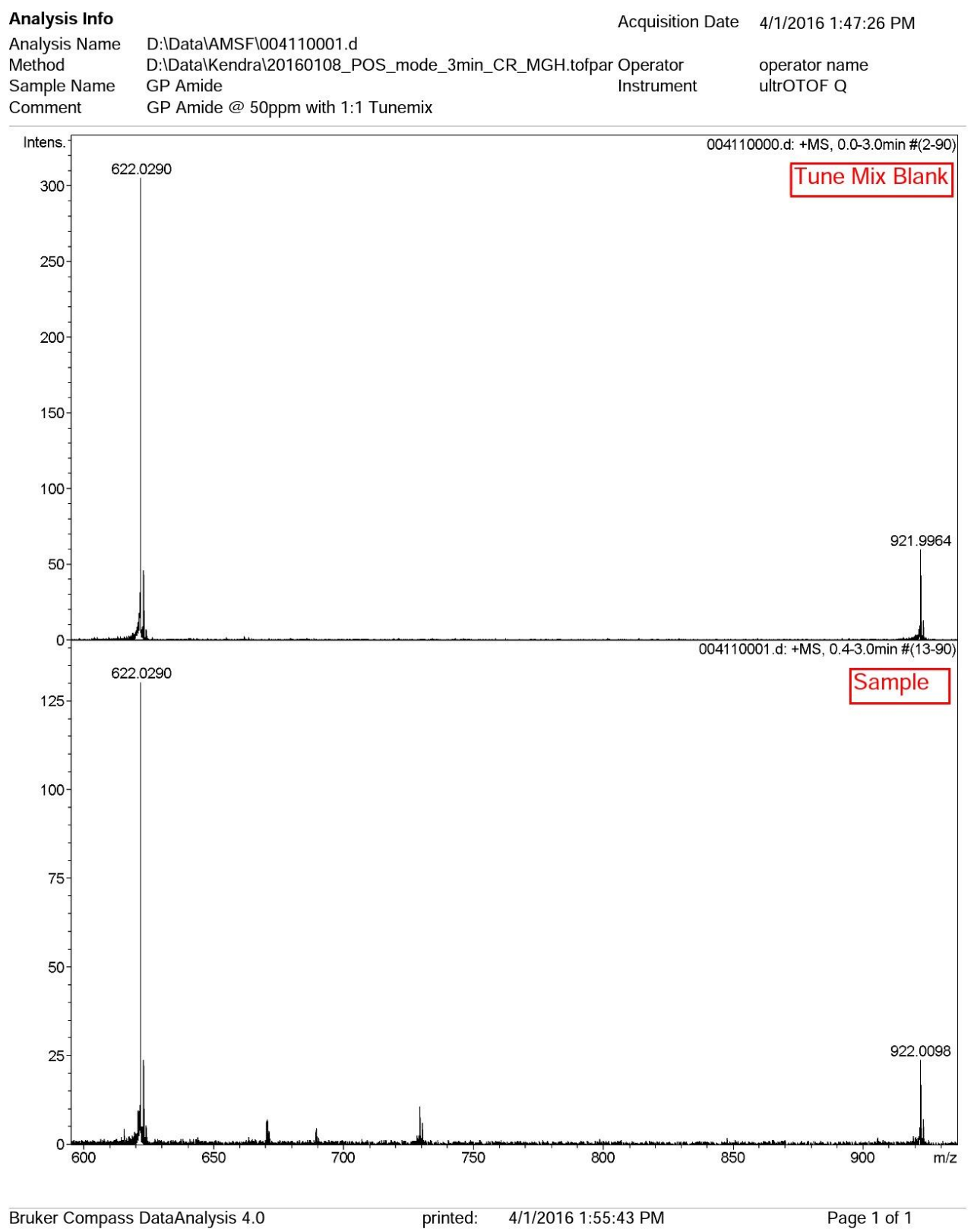


HRMS Report 2: $N$-(3-1H-imidazol-1-yl)-2-bromo-2-methylpropanamide

11 $\begin{aligned} & \text { FLORIDA } \\ & \text { INTERNATIONAL } \\ & \text { UNIVERSITY }\end{aligned}$

\title{
Advanced Mass Spectrometry Facility
}

\author{
Sample Analysis Report
}

\begin{abstract}
Report Date: $\quad$ April 7, 2016
Request date: $\quad$ April 5, 2016

Request description: One sample (Br Amide) was submitted for TOF HRMS analysis by Gloria Perez on 2016-4-5.

Report contents:

1. + ESI MS Report Summary

2. Submission document

3. Obtained TOF +ESI HRMS spectrum Tune Mixture Blank Br Amide m/z 200-1000

4. Obtained TOF +ESI HRMS spectrum Tune Mixture Blank and Br Amide m/z 250-350

5. Obtained TOF +ESI HRMS spectrum Tune Mixture Blank, Br Amide and theoretical isotope pattern $[\mathrm{M}+\mathrm{H}]^{+} \mathrm{m} / \mathrm{z} 270-280$

Methods: The sample was diluted to 20ppm in methanol and then mixed 1:1 with Tune Mixture and directly infused/analyzed by (+) ESI mode on an ultrOTOF-Q instrument.

Report summary: The ion observed at 274.0531 has a mass error of $6.9 \mathrm{ppm}$ when compared to the theoretical $[\mathrm{M}+\mathrm{H}]^{+}$ion of the provided formula $\mathrm{C}_{10} \mathrm{H}_{16} \mathrm{BrN}_{3} \mathrm{O}$. The isotope pattern is in good agreement with the theoretical.
\end{abstract}

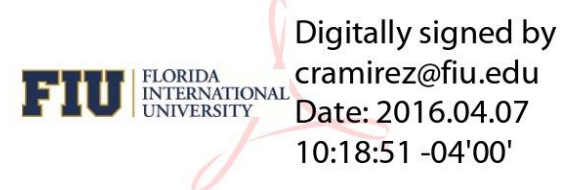

Advanced Mass Spectrometry Facility

Department of Chemistry and Biochemistry. 11200 SW $8^{\text {th }}$ ST. Room CP-174 Miami, FL. 33199.

Phone: 305.348.6656 - Fax: 305.348.3772 - E-mail: cramirez@fiu.edu 


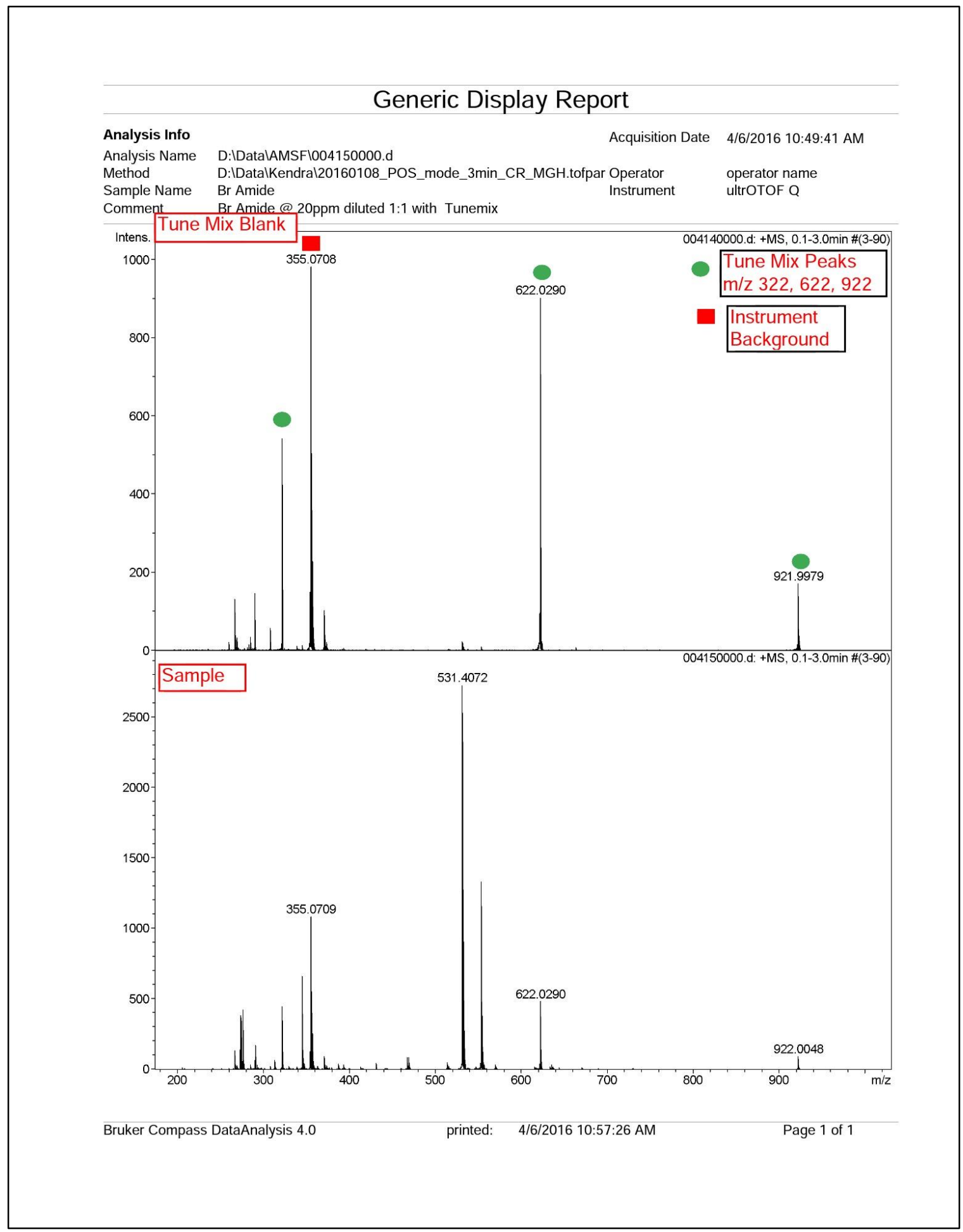




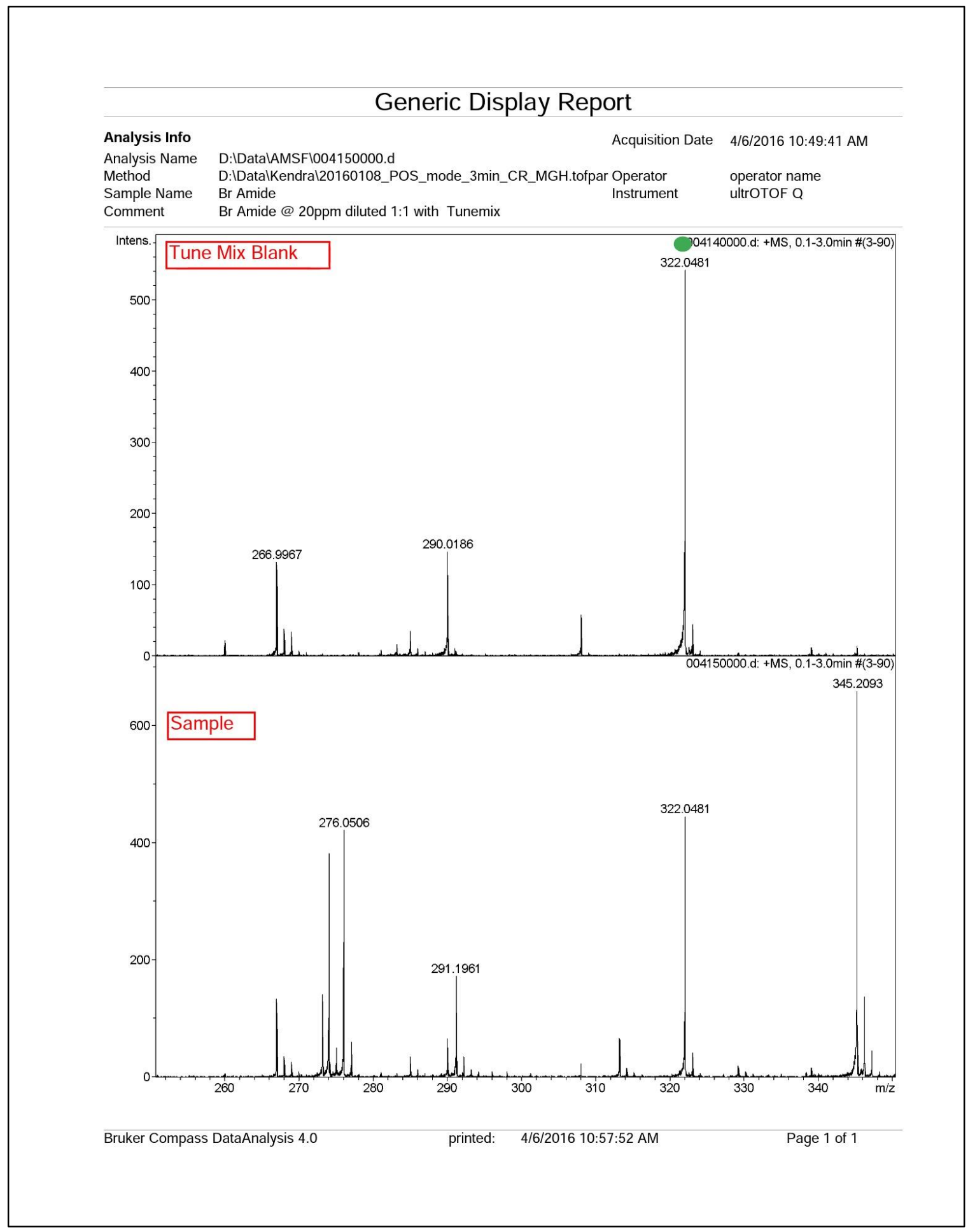




\section{Generic Display Report}

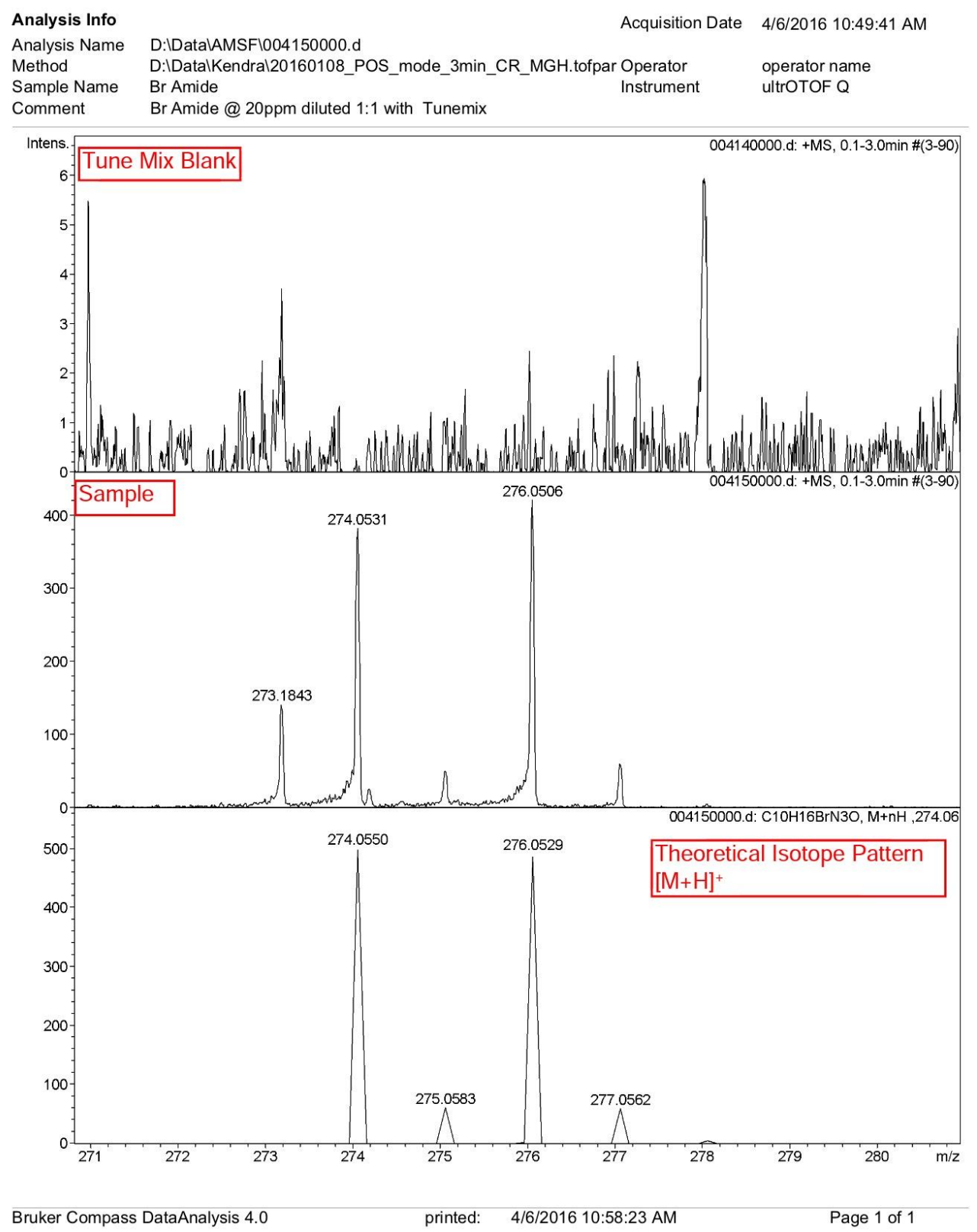




\section{REFERENCES}

(1) Murphy, M. P. Biochem. J. 2009, 417, 1.

(2) Cadenas, E.; Davies, K. J. A. Free Radical Biol. Med. 2000, 29, 222.

(3) Uttara, B.; Singh, A. V.; Zamboni, P.; Mahajan, R. T. Curr. Neuropharmacol. 2009, 7,65 .

(4) Halliwell, B. Am. J. Med. 1991, 91, 14S.

(5) Szeto, H. H. AAPS J. 2006, 8, E521.

(6) Smith, R. A. J.; Murphy, M. P. Discov Med. 2011, 11, 106.

(7) Oyewole, A. O.; Birch-Machin, M. A. FASEB J. 2015, 29, 4766.

(8) Paz, M. L.; Gonzalez Maglio, D. H.; Weill, F. S.; Bustamante, J.; Leoni, J. Photodermatol., Photoimmunol. Photomed. 2008, 24, 115.

(9) Scheffler, I. E. Biology Book 2007, 2, 492.

(10) Andersen, J. K. Nat Med 2004, 10 Suppl, S18.

(11) Klotz, L.-O. Biol. Chem. 2002, 383, 443.

(12) Fontanesi, F.; Soto, I. C.; Horn, D.; Barrientos, A. Am. J. Physiol. 2006, 291, C1129.

(13) Murphy, M. P. Free Radical Biol. Med. 2014, 66, 20.

(14) Halliwell, B. J. Neurochem. 2006, 97, 1634.

(15) Finkel, T. Nat. Rev. Mol. Cell Biol. 2005, 6, 971.

(16) Floyd, R. A.; Castro Faria Neto, H. C.; Zimmerman, G. A.; Hensley, K.; Towner, R. A. Free Radical Biol. Med. 2013, 62, 145.

(17) Pryor, W. A. Environ Health Perspect 1997, 105 Suppl 4, 875.

(18) Sun, Y. Free Radical Biol. Med. 1990, 8, 583.

(19) Frenkel, K. Pharmacol. Ther. 1992, 53, 127.

(20) Kensler, T. W.; Trush, M. A. Environ. Mutagen. 1984, 6, 593.

(21) James, A. M.; Cocheme, H. M.; Smith, R. A. J.; Murphy, M. P. J. Biol. Chem. 2005, $280,21295$.

(22) Murphy, M. P.; Smith, R. A. J. Annu. Rev. Pharmacol. Toxicol. 2007, 47, 629. 
(23) Slemmer, J. E.; Shacka, J. J.; Sweeney, M. I.; Weber, J. T. Curr. Med. Chem. 2008, 15,404 .

(24) Hoye, A. T.; Davoren, J. E.; Wipf, P.; Fink, M. P.; Kagan, V. E. Accounts of Chemical Research 2008, 41, 87.

(25) Firuzi, O.; Miri, R.; Tavakkoli, M.; Saso, L. Curr. Med. Chem. 2011, 18, 3871.

(26) Miller, E. R., III; Pastor-Barriuso, R.; Dalal, D.; Riemersma, R. A.; Appel, J. J.; Guallar, E. Ann. Intern. Med. 2005, 142, 37.

(27) Demura, M.; Kamo, N.; Kobatake, Y. Biochim. Biophys. Acta, Biomembr. 1985, 820, 207.

(28) Flewelling, R. F.; Hubbell, W. L. Biophys. J. 1986, 49, 531.

(29) Ross, M. F.; Kelso, G. F.; Blaikie, F. H.; James, A. M.; Cocheme, H. M.; Filipovska, A.; Ros, T.; Hurd, T. R.; Smith, R. A. J.; Murphy, M. P. Biochemistry (Moscow) 2005, 70, 222.

(30) Honig, B. H.; Hubbell, W. L.; Flewelling, R. F. Annu. Rev. Biophys. Biophys. Chem. 1986, 15, 163.

(31) Reddy, P. H. J. Biomed. Biotechnol. 2006, No pp. given.

(32) L., C. F. In Annu. Rev. Biochem. 1977, p 439.

(33) Kagan, V. E.; Serbinova, E. A.; Stoyanovsky, D. A.; Khwaja, S.; Packer, L. Methods Enzymol. 1994, 234, 343.

(34) Maguire, J. J.; Wilson, D. S.; Packer, L. J. Biol. Chem. 1989, 264, 21462.

(35) Ernster, L.; Forsmark, P.; Nordenbrand, K. BioFactors 1992, 3, 241.

(36) Asin-Cayuela, J.; Manas, A.-R. B.; James, A. M.; Smith, R. A. J.; Murphy, M. P. FEBS Lett. 2004, 571, 9.

(37) Sano, M.; Ernesto, C.; Thomas, R. G.; Klauber, M. R.; Schafer, K.; Grundman, M.; Woodbury, P.; Growdon, J.; Cotman, C. W.; Pfeiffer, E.; Schneider, L. S.; Thal, L. J. N Engl J Med 1997, 336, 1216.

(38) Jauslin, M. L.; Meier, T.; Smith, R. A. J.; Murphy, M. P. FASEB J. 2003, 17, 1972.

(39) Adlam, V. J.; Harrison, J. C.; Porteous, C. M.; James, A. M.; Smith, R. A. J.; Murphy, M. P.; Sammut, I. A. FASEB J. 2005, 19, 1088.

(40) Siler-Marsiglio, K. I.; Pan, Q.; Paiva, M.; Madorsky, I.; Khurana, N. C.; Heaton, M. B. Brain Res. 2005, 1052, 202. 
(41) Covey, M. V.; Murphy, M. P.; Hobbs, C. E.; Smith, R. A. J.; Oorschot, D. E. Exp. Neurol. 2006, 199, 513.

(42) Sheu, S.-S.; Nauduri, D.; Anders, M. W. Biochim. Biophys. Acta, Mol. Basis Dis. 2006, 1762, 256.

(43) Chang, H. Y.; Yang, X. Microbiol Mol Bio Rev 2000, 64, 821.

(44) Zhao, K.; Zhao, G.-M.; Wu, D.; Soong, Y.; Birk, A. V.; Schiller, P. W.; Szeto, H. H. J. Biol. Chem. 2004, 279, 34682.

(45) Szeto, H. H. AAPS J. 2006, 8, E277.

(46) Winterbourn, C. C.; Parsons-Mair, H. N.; Gebicki, S.; Gebicki, J. M.; Davies, M. J. Biochem. J. 2004, 381, 241.

(47) Zhao, K.; Luo, G.; Zhao, G.-M.; Schiller, P. W.; Szeto, H. H. J. Pharmacol. Exp. Ther. 2003, 304, 425.

(48) Zhao, K.; Luo, G.; Giannelli, S.; Szeto, H. H. Biochem. Pharmacol. 2005, 70, 1796.

(49) Wu, D.; Soong, Y.; Zhao, G.-M.; Szeto, H. H. Am. J. Physiol. 2002, 283, H783.

(50) Cho, J.; Won, K.; Wu, D.; Soong, Y.; Liu, S.; Szeto, H. H.; Hong, M. K. Coron Artery Dis 2007, 18, 215.

(51) Fangour, S.; Marini, M.; Good, J.; McQuaker, S. J.; Shiels, P. G.; Hartley, R. C. Age (Dordrecht, Neth.) 2009, 31, 269.

(52) Villamena, F. A.; Das, A.; Nash, K. M. Future Med. Chem. 2012, 4, 1171.

(53) Thornalley, P. J. Life Chem. Rep. 1986, 4, 57.

(54) Maples, K. R.; Green, A. R.; Floyd, R. A. CNS Drugs 2004, 18, 1071.

(55) Lapchak, P. A.; Schubert, D. R.; Maher, P. A. Transl. Stroke Res. 2011, 2, 209.

(56) Zuo, L.; Chen, Y.-R.; Reyes, L. A.; Lee, H.-L.; Chen, C.-L.; Villamena, F. A.; Zweier, J. L. J. Pharmacol. Exp. Ther. 2009, 329, 515.

(57) Isaev, N. K.; Novikova, S. V.; Stelmashook, E. V.; Barskov, I. V.; Silachev, D. N.; Khaspekov, L. G.; Skulachev, V. P.; Zorov, D. B. Biochemistry (Moscow) 2012, $77,996$.

(58) Becker, D. A.; Natero, R.; Echegoyen, L.; Lawson, R. C. J. Chem. Soc., Perkin Trans. 2 1998, 1289.

(59) Buettner, G. R. Arch. Biochem. Biophys. 1993, 300, 535. 
(60) Becker, D. A. J Am Chem Soc 1996, 118, 905.

(61) Kolhe, A., 2009.

(62) Becker, D. A.; Ley, J. J.; Echegoyen, L.; Alvarado, R. J. Am. Chem. Soc. 2002, 124, 4678 .

(63) Boyd, G. V. Journal of the Chemical Society (Resumed) 1959, 55.

(64) Becker, D. A.; Tamrakar, R.; The Florida International University, USA . 2013, p $78 \mathrm{pp}$.

(65) Marco Contelles, J. L.; Alcazar Gonzalez, A.; Consejo Superior de Investigaciones Cientificas CSIC , Spain; Fundacion para la Investigacion Biomedica del Hospital Universitario Ramon y Cajal . 2014, p 44pp.; Chemical Indexing Equivalent to 162:184333 (ES).

(66) Villamena, F. A.; Xia, S.; Merle, J. K.; Lauricella, R.; Tuccio, B.; Hadad, C. M.; Zweier, J. L. J Am Chem Soc 2007, 129, 8177.

(67) Romanet, R. F.; Burns, P. A.; Fischer, S. M.; Spara, P. P.; Balasubramanian, R. P.; Lincoln, D. G.; Kapp, D. L.; Eastman Kodak Company, USA . 1999, p 25 pp.

(68) Montalbetti, C. A. G. N.; Falque, V. Tetrahedron 2005, 61, 10827.

(69) Guo, Z.; Dowdy, E. D.; Li, W. S.; Polniaszek, R.; Delaney, E. Tetrahedron Lett. 2001, 42, 1843.

(70) Kornblum, N.; Blackwood, R. K.; Powers, J. W. J. Am. Chem. Soc. 1957, 79, 2507.

(71) Fujii, S.; Yamashita, S.; Kakigi, Y.; Aono, K.; Hamasaki, S.; Yusa, S.-i.; Nakamura, Y. Colloid Polym. Sci. 2013, 291, 1171.

(72) Kende, A. S.; Mendoza, J. S. Tetrahedron Lett. 1991, 32, 1699.

(73) Ito, M.; Okui, H.; Nakagawa, H.; Mio, S.; Iwasaki, T.; Iwabuchi, J. Heterocycles 2002, 57,881 .

(74) Sacher, J. R., 2012.

(75) Klivenyi, P.; Matthews, R. T.; Wermer, M.; Yang, L.; MacGarvey, U.; Becker, D. A.; Natero, R.; Beal, M. F. Exp. Neurol. 1998, 152, 163.

(76) Matias, A. C.; Biazolla, G.; Cerchiaro, G.; Keppler, A. F. Bioorg. Med. Chem. 2016, $24,232$.

(77) Kerber, R. C.; Urry, G. W.; Kornblum, N. J. Am. Chem. Soc. 1965, 87, 4520. 
